MANCHESTER

1824

\title{
Economics
}

Discussion Paper Series

EDP-1314

\section{Impossibility Results for Parametrized Notions of Efficiency and Strategy- proofness in Exchange Economies}

\author{
Wonki Jo Cho
}

September 2013

Economics

School of Social Sciences

The University of Manchester

Manchester M13 9PL 


\title{
Impossibility Results for Parametrized Notions of Efficiency and Strategy-proofness in Exchange Economies
}

\author{
Wonki Jo Cho*
}

July 10, 2012

\begin{abstract}
We study a standard model of exchange economies with individual endowments. It is well known that no rule is individually rational, efficient, and strategyproof. In order to quantify the extent of this impossibility, we parametrize axioms on allocation rules. Given an axiom $A$, a parametrization of $A$ is a continuum of axioms $\{\delta-A\}_{\delta \in[0,1]}$ such that (i) $\delta$ - $A$ is equivalent to $A$ only if $\delta=1$; (ii) $\delta$ - $A$ is vacuous only if $\delta=0$; and (iii) for each pair $\delta, \delta^{\prime} \in[0,1]$ with $\delta<\delta^{\prime}, \delta^{\prime}-A$ implies $\delta$ - $A$. Thus, as $\delta$ decreases from 1 to $0, \delta$ - $A$ weakens monotonically, eventually to a vacuous requirement. We consider two parametrizations $\{\delta \text {-efficiency }\}_{\delta \in[0,1]}$ and $\{\delta \text {-strategy-proofness }\}_{\delta \in[0,1]}$, and investigate their compatibility with individual rationality for the class of two-agent economies defined on a domain containing linear preference relations. We show that (i) for each $\delta \in(0,1]$, no rule is individually rational, $\delta$-efficient, and strategy-proof; and (ii) for each $\delta \in(0,1]$, no rule is individually rational, efficient, and $\delta$-strategy-proof. These results strengthen existing impossibility theorems in two directions that have not been explored so far.

Journal of Economic Literature Classification Numbers: D71

Key Words: exchange economy, individual rationality, $\delta$-efficiency, $\delta$-strategyproofness.
\end{abstract}

${ }^{*}$ School of Social Sciences, University of Manchester, Oxford Road, Manchester M13 9PL, United Kingdom; jo.cho@manchester.ac.uk. I am deeply grateful to William Thomson for his guidance. 


\section{Introduction}

We study standard exchange economies with individual endowments. There is a set of perfectly divisible commodities. Each agent has a preference relation defined over nonnegative amounts of those commodities. He also owns some amounts of the commodities, which we call an (individual) endowment. An economy is a profile of preference relations and endowments, and an allocation for the economy is a profile of (consumption) bundles whose sum is equal to the sum of the endowments. An (allocation) rule assigns to each economy an allocation for it.

Our objective is to search for rules satisfying some desirable properties, or axioms, and the following three axioms have long dominated the literature on this quest: (i) individual rationality, the requirement that for each economy, a rule assign to each agent a bundle that he finds at least as desirable as his endowment; (ii) efficiency, the requirement that for each economy, a rule select an allocation such that no other allocation Pareto dominates it; and (iii) strategy-proofness, the requirement that a rule select allocations in such a way that no agent ever benefits from lying about his preference relation.

The three requirements, however, are incompatible. Hurwicz (1972) shows that for the class of two-agent and two-commodity economies, no rule meets all of them. ${ }^{1}$ Subsequent studies strengthen this theorem mainly in two directions. 2 The first strengthening involves establishing similar results on smaller preference domains. Hurwicz (1972) works with classical preference relations (i.e., those that are continuous, monotone, and strictly convex). As it turns out, this is quite a rich domain, and one can derive his impossibility theorem with a much smaller subset of preference relations. Parallel results are available on the domain of linear preference relations (Schummer, 1997) and the domain of CES preference relations (Ju, 2003).

The second way of strengthening is to show that each efficient and strategy-proof rule violates some fairness axiom, and then obtain Hurwicz's (1972) theorem as a corollary. A number of papers consider the following as fairness criteria: (i) non-dictatorship,

\footnotetext{
${ }^{1}$ Hurwicz's (1972) theorem covers only two-agent and two-commodity economies, and Serizawa (2002) generalizes it to economies with an arbitrary number of agents and an arbitrary number of commodities.

${ }^{2}$ Most papers cited here pursue the two directions simultaneously.
} 
the requirement that there be no agent who receives everything for each economy (Dasgupta, Hammond, and Maskin, 1979; Ju, 2003; and Schummer, 1997); (ii) non-inversedictatorship, the requirement that there be no agent who receives nothing for each economy (Zhou, 1991); and (iii) minimum consumption guarantee, the requirement that each agent receive a bundle bounded away from the origin (Serizawa and Weymark, 2003).

Among this range of stronger impossibility results, absent is a theorem that weakens efficiency or strategy-proofness while maintaining individual rationality ${ }^{3}$ We attribute the absence to two sources. The first relates to the indisputable normative appeal of efficiency and strategy-proofness. Viewed separately, efficiency is so mild a requirement that weakening it appears hardly necessary (no economist would object to making one agent better off without hurting any other agent). On the other hand, strategyproofness, though demanding, is an axiom that we cannot dispense with in the context where agents' private information, e.g., preference relations, should be elicited. On these grounds, the two axioms are widely accepted, to the extent that most axiomatic analyses take them as "basic" requirements and study the consequences of imposing some other axioms additionally.

The inherent difficulty in weakening efficiency or strategy-proofness has also played an important role. When a rule violates efficiency, it is not trivial to quantify the extent of that violation. On the other hand, if a rule is not strategy-proof, it is often subjected to the following test: in "how many" economies can an agent profitably misreport his private information? ${ }^{4}$ While this is one way of quantifying the degree of manipulability, it does not measure how much an agent can gain with strategic behavior.

Our motivation to weaken efficiency and strategy-proofness goes beyond theoretical interest and is based on the following scenario we model. A group of agents, each with an endowment, gather to find an allocation that is beneficial to all. The endowments are privately owned, and we operationalize this notion of private ownership by giving each agent the right to consume his endowment if he so desires. Then as the agents negotiate on who gets what, their endowments serve as a critical benchmark: whenever

\footnotetext{
${ }^{3}$ When the preference domain on which a rule is defined becomes smaller, the scope, and hence strength, of strategy-proofness decreases. However, even on the smaller domain, the spirit of strategyproofness that no agent ever benefits from lying about his preference relation, remains the same.

${ }^{4}$ E.g., Aleskerov and Kurbanov (1999), Kelly (1993), Maus, Peters, and Storcken (2007a, 2007b), and Smith (1999).
} 
the collective decision assigns an agent a bundle less desirable than his endowment, he can simply walk out. In situations like this, individual rationality is an axiom that should be met at all costs, and Hurwicz (1972) suggests that we cannot have both efficiency and strategy-proofness. Then how much of efficiency should we sacrifice to have an individually rational and strategy-proof rule? Or how much of strategy-proofness should we abandon to have an individually rational and efficient rule? These are the questions we address.

The contribution of this paper consists in (i) providing weakenings of efficiency and strategy-proofness - in fact, parametrizations thereof; and (ii) showing that when combined with individual rationality, either of the two axioms forces a rule to satisfy only the vacuous version of the remaining axiom. Before introducing our parametrizations, let us first explain an underlying principle. Let $A$ be an axiom. Let $[0,1]$ be the parameter space and $\delta$ the parameter. A parametrization of $A$ is a continuum of axioms $\{\delta-A\}_{\delta \in[0,1]}$ such that (i) $\delta-A$ is equivalent to $A$ only if $\delta=1$; (ii) $\delta$ - $A$ is vacuous only if $\delta=0$; and (iii) for each pair $\delta, \delta^{\prime} \in[0,1]$ with $\delta<\delta^{\prime}, \delta^{\prime}-A$ implies $\delta$ - $A$. In short, decreasing $\delta$ from 1 to 0 weakens $A$ monotonically, eventually to a vacuous requirement. Our parametrizations of efficiency and strategy-proofness are in line with this spirit, and in order to weaken the axioms monotonically, we use the Hausdorff distance in the Euclidean space.

More specifically, the parametrization of efficiency is obtained by the following procedure. Given an economy, normalize to one the Hausdorff distance (induced by the standard Euclidean distance) between the set of efficient allocations and the set of feasible allocations.$^{5}$ For each $\delta \in[0,1]$, an allocation is $\delta$-efficient if the normalized distance between the allocation and the set of efficient allocations is $1-\delta$. As $\delta$ decreases from 1 to 0 , the set of $\delta$-efficient allocations expands monotonically, eventually coinciding with the set of feasible allocations. A rule is $\delta$-efficient if for each economy, it selects a $\delta$-efficient allocation.

Next, to illustrate the parametrization of strategy-proofness, let $\delta \in[0,1]$. Fix an economy and an agent. Normalize to one the Hausdorff distance between (i) the

\footnotetext{
${ }^{5}$ Our definition of feasibility requires that the sum of bundles equals the sum of endowments. Since we work with strictly monotone preference relations, the latter definition allows us to ignore those uninteresting allocations that waste some of endowments.
} 
set of feasible bundles; and (ii) the intersection of the set of feasible bundles and the lower contour set of his true preference relation at the bundle he receives by telling the truth. Then $\delta$-strategy-proofness requires that each bundle he can obtain with misrepresentation lie within the normalized distance $1-\delta$ of set (ii). As $\delta$ decreases from 1 to 0 , the set of bundles that the agent can receive by lying expands monotonically, and when $\delta=0, \delta$-strategy-proofness places no restriction.

Our parametrizations enable us to measure the "degree" of incompatibility of individual rationality, efficiency, and strategy-proofness. In light of Hurwicz's (1972) theorem, one may expect that for $\delta \in[0,1)$ sufficiently close to 1 , (i) no rule is individual rational, $\delta$-efficient, and strategy-proof; and (ii) no rule is individual rational, efficient, and $\delta$-strategy-proof. But what we show is much stronger than these conjectures. We establish that for the class of two-agent economies defined on a domain containing linear preference relations, for each $\delta \in(0,1]$, statements (i) and (ii) above are true (Theorems 1 and 2, respectively). The remaining case of $\delta=0$ is an exception. For (i), the no-trade rule, namely the rule that for each economy, selects the endowment profile as an allocation, satisfies the three axioms; and for (ii), any rule that for each economy, selects an individually rational and efficient allocation, satisfies the three axioms.

One may criticize our parametrizations, saying that the expansion process used to define $\delta$-efficiency and $\delta$-strategy-proofness have little welfare content in it. For instance, there is an economy with, say, a 0.3-efficient allocation that Pareto dominates a 0.6-efficient allocation; and an agent may benefit more greatly by manipulating a 0.3-strategy-proof rule than by manipulating a 0.6-strategy-proof rule. Such criticism would be warranted if we compared the 0.3-efficient and 0.6-efficient allocations and announced, say, that the latter is more desirable than the former; or if we argued that the 0.6-strategy-proof rule is less vulnerable to strategic misrepresentation than the 0.3-strategy-proof rule is. But we make no comparison of this kind, nor is it our focus. Rather, our focus is on weakening efficiency and strategy-proofness to the extent that each of them reduces to a near-vacuous requirement, and showing that even in such extreme case, they remain incompatible with other axioms.

Further, as $\delta$ approaches zero, the Euclidean distance plays an increasingly minor role in our parametrizations. Since preference relations are continuous, for $\delta \in(0,1]$ 
sufficiently close to zero, almost all feasible allocations, and hence almost all profiles of feasible welfare levels, are considered $\delta$-efficient; similarly, $\delta$-strategy-proofness allows an agent to attain almost all feasible bundles, and hence almost all feasible welfare levels, by manipulating a rule. These observations suggest that the Euclidean distance is not the main driving factor in our impossibility results. Motivated by the latter intuition, we check whether our results are robust to changes in the distance notion in the Euclidean space or the parametrization method.

Concerning the issue of using different distance notions, Theorem 1 remains true even if an arbitrary distance is used to define $\delta$-efficiency; and Theorem 2 holds as long as a distance satisfying a mild requirement is used to define $\delta$-strategy-proofness. Similarly, the two theorems continue to hold if we adopt different parametrization methods. We provide conditions that reasonable parametrizations of efficiency and strategy-proofness should satisfy, and then show that similar results follow under those conditions (Theorems 3 and 4 ).

The rest of the paper proceeds as follows. We set up the model in Section 2 and introduce axioms on rules in Section 3. Our impossibility results are in Section 4, and we check their robustness in Section 5 .

\section{The Model}

Let $\boldsymbol{N} \equiv\{1, \cdots, n\}$ be the set of agents and $\boldsymbol{M} \equiv\{1, \cdots, m\}$ the set of commodities. For each $i \in N$, agent $i$ has (i) a continuous, strictly monotone, ${ }^{6}$ and convex preference relation $\boldsymbol{R}_{\boldsymbol{i}}$ on $\mathbb{R}_{+}^{M}$; and (ii) an endowment $\boldsymbol{\omega}_{\boldsymbol{i}} \equiv\left(\omega_{i 1}, \cdots, \omega_{i m}\right) \in \mathbb{R}_{+}^{M}$. Denote by $\boldsymbol{P}_{\boldsymbol{i}}$ and $\boldsymbol{I}_{\boldsymbol{i}}$ the strict preference and indifference relations, respectively, associated with $R_{i}$. Let $\mathcal{R}$ be a domain of admissible preference relations on $\mathbb{R}_{+}^{M}$. Let $\boldsymbol{R} \equiv\left(R_{i}\right)_{i \in N}$ be the preference profile and $\boldsymbol{\omega} \equiv\left(\omega_{i}\right)_{i \in N}$ the endowment profile.

We are primarily concerned with linear preference relations, i.e., those that can be represented by linear functions. Let $\boldsymbol{\mathcal { R }}_{\text {lin }}$ be the domain of linear preference relations. For each $R_{0} \in \mathcal{R}_{\text {lin }}$, all relevant information about $R_{0}$ is captured by a vector $\boldsymbol{r}_{\mathbf{0}} \in$

\footnotetext{
${ }^{6} \mathrm{~A}$ preference relation $R_{i}$ is strictly monotone if for each pair $x_{i}, y_{i} \in \mathbb{R}_{+}^{M}$ such that for each $\ell \in M$, $x_{i \ell} \geq y_{i \ell}$, with at least one strict inequality, $x_{i} P_{i} y_{i}$.
} 
$\mathbb{R}_{+}^{M}$ that is normal to indifference curves (surfaces) of $\left.R_{0} \cdot\right]^{7}$ When there are only two commodities, i.e., $m=2$, we simply take the common slope of indifference curves of $R_{0}$ and denote its absolute value by $\boldsymbol{s}\left(\boldsymbol{R}_{\mathbf{0}}\right)$.

An economy is a list $(R, \omega) \in \mathcal{R}^{N} \times \mathbb{R}_{+}^{M N}$. Let $\mathcal{E}(\mathcal{R})$ be the collection of all economies in which each agent has a preference relation in $\mathcal{R}$. Given an endowment profile $\omega \in \mathbb{R}_{+}^{M N}$, a (feasible) allocation is a profile $x \equiv\left(x_{i}\right)_{i \in N} \in \mathbb{R}_{+}^{M N}$ such that (i) for each $i \in N, x_{i} \equiv\left(x_{i 1}, \cdots, x_{i m}\right) \in \mathbb{R}_{+}^{M}$; and (ii) for each $\ell \in M, \sum_{i \in N} x_{i \ell}=\sum_{i \in N} \omega_{i \ell}$. For each $i \in N$, we call $x_{i}$ agent $i$ 's (consumption) bundle. Let $\boldsymbol{Z}(\boldsymbol{\omega})$ be the set of all feasible allocations. For each $i \in N$, let $\boldsymbol{Z}_{\boldsymbol{i}}(\boldsymbol{\omega}) \equiv\left\{x_{i} \in \mathbb{R}_{+}^{M}\right.$ : for each $\ell \in$ $\left.M, x_{i \ell} \leq \sum_{j \in N} \omega_{j \ell}\right\}$ be the projection of $Z(\omega)$ onto agent $i$ 's consumption space $\mathbb{R}_{+}^{M}$. An (allocation) rule, denoted by $\boldsymbol{\varphi}$, is a mapping from $\mathcal{E}(\mathcal{R})$ to $\mathbb{R}_{+}^{M N}$ such that for each $(R, \omega) \in \mathcal{E}(\mathcal{R}), \varphi(R, \omega) \in Z(\omega)$. For each $(R, \omega) \in \mathcal{E}(\mathcal{R})$ and each $i \in N$, denote agent $i$ 's bundle in $\varphi(R, \omega)$ by $\varphi_{i}(R, \omega)$.

Note that we require equality in the definition of feasibility. To explain why we use this formulation, consider a profile of bundles whose sum is less than the sum of endowments. Since we work with strictly monotone preference relations, we can always make the agents better off by distributing the remaining amount. Consequently, as agents negotiate on allocation of the endowments, the proposed profile of bundles is not renegotiation-proof. In this regard, such profiles of bundles are not appealing, and our formulation of feasibility allows us to ignore them from the outset (for consequences of this formulation, see Footnote 10.

Finally, we use the following notation. For each $R_{0} \in \mathcal{R}$ and each $x_{0} \in \mathbb{R}_{+}^{M}$, let $\boldsymbol{L}\left(\boldsymbol{R}_{\mathbf{0}}, \boldsymbol{x}_{\mathbf{0}}\right) \equiv\left\{y_{0} \in \mathbb{R}_{+}^{M}: x_{0} R_{0} y_{0}\right\}$ be the lower contour set of $R_{0}$ at $x_{0}$. Let $\|\cdot\|$ be the Euclidean distance on $\mathbb{R}_{+}^{M N}$; i.e., for each pair $a, b \in \mathbb{R}_{+}^{M N},\|a-b\| \equiv$ $\left[\sum_{i \in N, \ell \in M}\left(a_{i \ell}-b_{i \ell}\right)^{2}\right]^{1 / 2}$. Given a point $a \in \mathbb{R}_{+}^{M N}$ and a non-empty set $B \subseteq \mathbb{R}_{+}^{M N}$, let $\boldsymbol{d}(\boldsymbol{a}, \boldsymbol{B}) \equiv \inf _{b \in B}\|a-b\|$. Given non-empty sets $A, B \subseteq \mathbb{R}_{+}^{M N}$, let $\boldsymbol{d}(\boldsymbol{A}, \boldsymbol{B}) \equiv$ $\max \left\{\sup _{a \in A} d(a, B), \sup _{b \in B} d(b, A)\right\}$. When applied to sets in $\mathbb{R}_{+}^{M N}, d(\cdot, \cdot)$ is the Hausdorff distance induced by the Euclidean distance $\|\cdot\|$. Similarly, we can define the distances between (i) a pair of points in $\mathbb{R}_{+}^{M}$; (ii) a point and a set in $\mathbb{R}_{+}^{M}$; and (iii) a pair of sets in $\mathbb{R}_{+}^{M}$. Denote these distances by $\|\cdot\|$ and $d(\cdot, \cdot)$ as well.

\footnotetext{
${ }^{7}$ Preference relations and bundles that are attached to no particular agent are denoted by $R_{0}$ and $x_{0}$, respectively.
} 
Also, given an arbitrary Euclidean space $\mathbb{R}_{+}^{k}$ and distinct points $a, b \in \mathbb{R}_{+}^{k}$, let $\operatorname{seg}[\boldsymbol{a}, \boldsymbol{b}] \equiv\{t a+(1-t) b: 0 \leq t \leq 1\}$ be the line segment connecting $a$ and $b$. Similarly, let $\operatorname{seg}(\boldsymbol{a}, \boldsymbol{b}] \equiv\{t a+(1-t) b: 0 \leq t<1\}$ and $\operatorname{seg}[\boldsymbol{a}, \boldsymbol{b}) \equiv\{t a+(1-t) b: 0<t \leq 1\}$. Given distinct points $a^{1}, \ldots, a^{h} \in \mathbb{R}_{+}^{k}$, denote by pol $\left[\boldsymbol{a}^{\mathbf{1}}, \cdots, \boldsymbol{a}^{\boldsymbol{h}}\right]$ the polygon whose sides are given by line segments $\operatorname{seg}\left[a^{1}, a^{2}\right], \operatorname{seg}\left[a^{2}, a^{3}\right], \cdots, \operatorname{seg}\left[a^{h-1}, a^{h}\right]$, and $\operatorname{seg}\left[a^{h}, a^{1}\right]$.

\section{Axioms on Allocation Rules}

In this section, we introduce axioms on rules. Our first axiom places a lower bound on agents' welfare. Given an economy, each agent is equipped with an endowment. Interpreting this as his private ownership, we are interested in allocations that respect it. Therefore, in order for an allocation to be desirable, it should assign each agent a bundle that he finds at least as preferable as his endowment. Formally, for each $(R, \omega) \in \mathcal{E}(\mathcal{R})$ and each $x \in Z(\omega), x$ is individually rational for $(\boldsymbol{R}, \boldsymbol{\omega})$ if for each $i \in N, x_{i} R_{i} \omega_{i}$. Let $\boldsymbol{I} \boldsymbol{R}(\boldsymbol{R}, \boldsymbol{\omega})$ be the set of all individually rational allocations for $(R, \omega)$. The following axiom requires that for each economy, a rule select an individually rational allocation.

Individual Rationality: For each $(R, \omega) \in \mathcal{E}(\mathcal{R}), \varphi(R, \omega)$ is individually rational for $(R, \omega)$.

To introduce our next axiom, we first define the notion of Pareto dominance. Let $(R, \omega) \in \mathcal{E}(\mathcal{R})$. For each pair $x, y \in Z(\omega), \boldsymbol{x}$ Pareto dominates $\boldsymbol{y}$ for $(\boldsymbol{R}, \boldsymbol{\omega})$ if (i) for each $i \in N, x_{i} R_{i} y_{i}$; and (ii) for some $j \in N, x_{j} P_{j} y_{j}$. For each $x \in Z(\omega), x$ is efficient for $(\boldsymbol{R}, \boldsymbol{\omega})$ if there is no $y \in Z(\omega)$ such that $y$ Pareto dominates $x$ for $(R, \omega)$. Let $\boldsymbol{E}(\boldsymbol{R}, \boldsymbol{\omega})$ be the set of all efficient allocations for $(R, \omega)$. The following axiom requires that for each economy, a rule select an efficient allocation.

Efficiency: For each $(R, \omega) \in \mathcal{E}(\mathcal{R}), \varphi(R, \omega)$ is efficient for $(R, \omega)$.

Now we introduce the notion of parametrization of an axiom. Let $A$ be an axiom. Let $[0,1]$ be the parameter space and $\delta$ the parameter. A parametrization of $\boldsymbol{A}$ (in terms of strength) is a continuum of axioms $\{\delta-A\}_{\delta \in[0,1]}$ such that (i) $\delta$ - $A$ is equivalent to $A$ only if $\delta=1$; (ii) $\delta$ - $A$ is vacuous only if $\delta=0$; and (iii) for each pair 


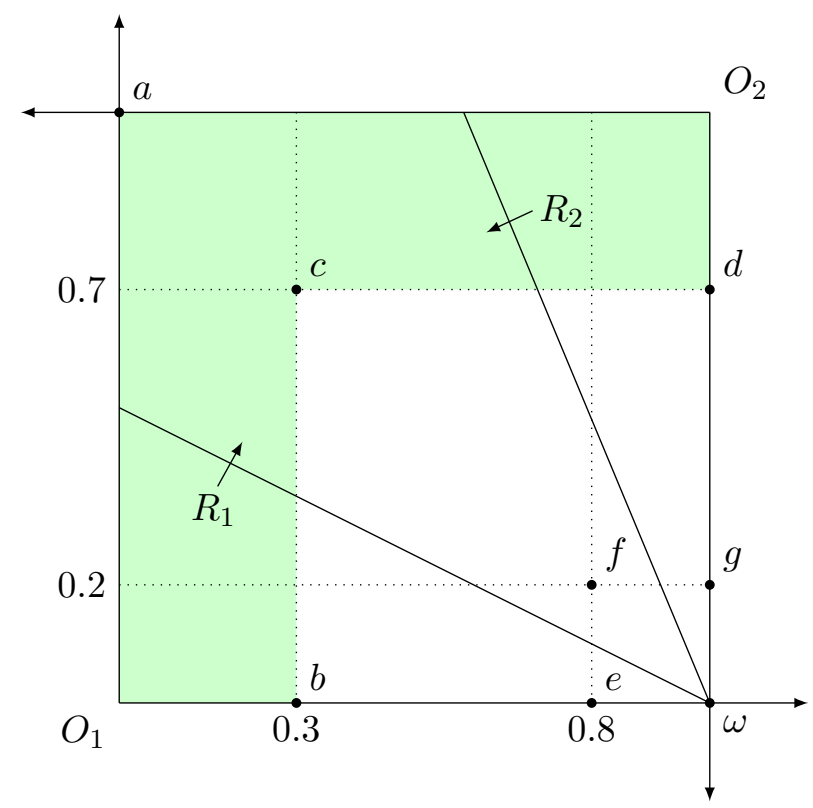

Figure 1: Illustration of $\boldsymbol{\delta}$-efficiency (Example 1). Let $N=\{1,2\}$ and $M=\{1,2\}$. For the economy $(R, \omega)$ as specified in the figure, $E(R, \omega)=\operatorname{seg}\left[O_{1}, a\right] \cup \operatorname{seg}\left[a, O_{2}\right]$. Also, $E^{0.7}(R, \omega)=$ $\operatorname{pol}\left[O_{1}, a, O_{2}, d, c, b\right]$ (shaded) and $E^{0.2}(R, \omega)=\operatorname{pol}\left[O_{1}, a, O_{2}, g, f, e\right]$. As $\delta \in(0,1)$ becomes smaller, $E^{\delta}(R, \omega)$ expands monotonically while maintaining a similar shape, and when $\delta=0$, it coincides with the set of feasible allocations.

$\delta, \delta^{\prime} \in[0,1]$ with $\delta<\delta^{\prime}, \delta^{\prime}-A$ implies $\delta$ - $A .^{8}$ In short, decreasing $\delta$ from 1 to 0 weakens $A$ monotonically, eventually to a vacuous requirement.

To parametrize efficiency, note that the axiom requires a rule to select from the set of efficient allocations. In other words, the Euclidean distance between the chosen allocation and the set of efficient allocations is zero. If a rule is not efficient, then the latter distance is positive, the maximum of which is the Hausdorff distance between the set of feasible allocations and the set of efficient allocations. Thus, one way of weakening efficiency is to allow a rule to choose an allocation that lies within a certain distance from the set of efficient allocations, relative to the maximum distance (as long as the maximum distance is positive).

\footnotetext{
${ }^{8}$ In a model where agents collectively own an endowment, Moulin and Thomson (1988) take a similar approach to weakening "individual rationality from equal division of the aggregate resources", the requirement that a rule assign to each agent a bundle that is at least as desirable as equal division of the collective endowment.
} 
Formally, let $\delta \in[0,1]$. For each $(R, \omega) \in \mathcal{E}(\mathcal{R})$ and each $x \in Z(\omega), x$ is $\delta$ efficient for $(\boldsymbol{R}, \boldsymbol{\omega})$ if $d(x, E(R, \omega)) \leq(1-\delta) d(Z(\omega), E(R, \omega)) .^{9}$ Let $\boldsymbol{E}^{\delta}(\boldsymbol{R}, \boldsymbol{\omega})$ be the set of all $\delta$-efficient allocations for $(R, \omega)$. As $\delta$ decreases from 1 to 0 , the set of $\delta$-efficient allocations expands monotonically, and when $\delta=0$, it covers the set of feasible allocations $\sqrt{10}^{10}$ The following axiom requires that for each economy, a rule select a $\delta$-efficient allocation.

$\delta$-Efficiency: For each $(R, \omega) \in \mathcal{E}(\mathcal{R}), \varphi(R, \omega)$ is $\delta$-efficient for $(R, \omega)$.

Example 1. Illustration of $\delta$-efficiency. Let $N=\{1,2\}$ and $M=\{1,2\}$. Let $\omega_{1}=$ $(1,0)$ and $\omega_{2}=(0,1)$. Let $R \in \mathcal{R}_{\text {lin }}^{N}$ be as specified in Figure 1. We use the Edgeworth box representation of allocations. First, $E(R, \omega)=\operatorname{seg}\left[O_{1}, a\right] \cup \operatorname{seg}\left[a, O_{2}\right]$. Since $\omega$ is the point in $Z(\omega)$ with the maximum distance from $E(R, \omega), d(Z(\omega), E(R, \omega))=$ $d(\omega, E(R, \omega))$. In order to obtain $E^{0.7}(R, \omega)$, for each $x \in E(R, \omega)$, take a closed ball of radius $0.3 d(\omega, E(R, \omega))$ centered at $x$, and then take the union of all those balls. This construction yields that $E^{0.7}(R, \omega)=\operatorname{pol}\left[O_{1}, a, O_{2}, d, c, b\right]$ (shaded). Similarly, $E^{0.2}(R, \omega)=\operatorname{pol}\left[O_{1}, a, O_{2}, g, f, e\right]$. As $\delta \in(0,1)$ becomes smaller, $E^{\delta}(R, \omega)$ expands monotonically while maintaining a similar shape. When $\delta=0, E^{\delta}(R, \omega)=Z(\omega)$ and $\delta$-efficiency has no bite.

Our last axiom pertains to the strategic behavior of agents. In many applications, agents' preference relations are private information and agents are not constrained to tell the truth. In fact, an agent may find it profitable to misrepresent his preference relation and manipulate the rule in his favor. Thus, we require that a rule be immune to such misrepresentation. Whatever the announcement of other agents are, no agent ever benefits from lying about his preference relation.

Strategy-proofness: For each $(R, \omega) \in \mathcal{E}(\mathcal{R})$, each $i \in N$, and each $R_{i}^{\prime} \in \mathcal{R}$, $\varphi_{i}(R, \omega) R_{i} \varphi_{i}\left(R_{i}^{\prime}, R_{-i}, \omega\right)$.

\footnotetext{
${ }^{9}$ If $\|\omega\|$ is small, even a slight expansion of the set of efficient allocations may contain the set of feasible allocations. Therefore, we use the distance $d(Z(\omega), E(R, \omega))$ as a normalizing factor so that $\delta$-efficiency properly factors in the "size" of an economy under consideration.

${ }^{10}$ Our definition of feasibility requires that the sum of bundles equal the sum of endowments. Therefore, if $\delta=0, E^{\delta}(R, \omega)$ coincides with the set of allocations that satisfy this condition, but it still excludes those that do not fully allocate the endowments among the agents. We believe that the latter allocations do not deserve much attention since we work with strictly monotone preference relations.
} 


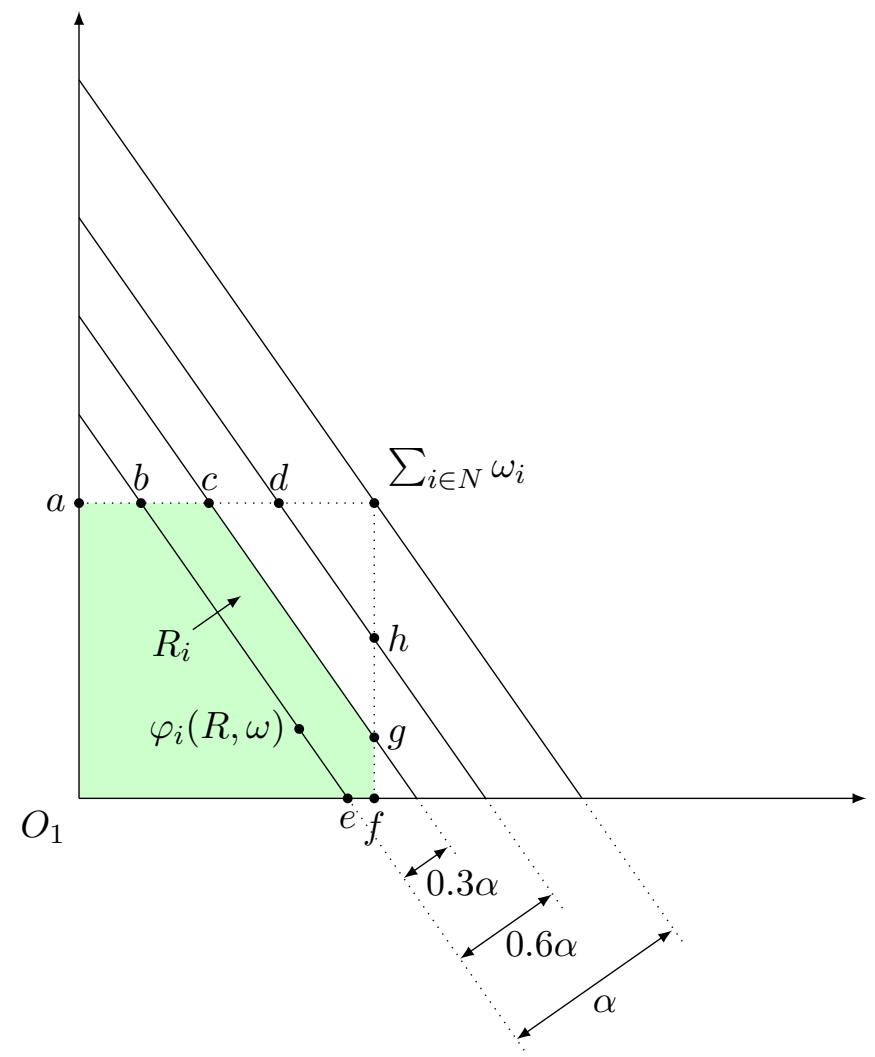

Figure 2: Illustration of $\boldsymbol{\delta}$-strategy-proofness (Example 2). Let $M=\{1,2\}$ and $(R, \omega) \in \mathcal{E}(\mathcal{R})$. Let $i \in N$, and suppose that $R_{i}$ and $\varphi_{i}(R, \omega)$ are as specified in the figure. Let $R_{i}^{\prime} \in \mathcal{R}$. Strategy-proofness requires that $\varphi_{i}\left(R_{i}^{\prime}, R_{-i}, \omega\right) \in \operatorname{pol}\left[O_{1}, a, b, e\right]$. By contrast, 0.7-strategy-proofness only requires that $\varphi_{i}\left(R_{i}^{\prime}, R_{-i}, \omega\right)$ lie within $0.3 \alpha$ of pol $\left[O_{1}, a, b, e\right]$; i.e., $\varphi_{i}\left(R_{i}^{\prime}, R_{-i}, \omega\right) \in$ pol $\left[O_{1}, a, c, g, f\right]$ (shaded). Similarly, 0.3-strategy-proofness requires that $\varphi_{i}\left(R_{i}^{\prime}, R_{-i}, \omega\right) \in \operatorname{pol}\left[O_{1}, a, d, h, f\right]$. If $\delta=0$, then $\delta$-strategy-proofness allows agent $i$ to obtain any feasible bundle with misrepresentation.

Note incidentally that our notion of misrepresentation is restricted to that of preference relations, excluding endowments. We can also imagine a situation where each agent's endowment is private information and a rule takes as an input whatever the agents submit as their endowments. This provides agents with an additional incentive to lie, where the lie can take various forms (e.g., withhold or destroy part of one's endowment; and borrow some amount of the commodities from the outside world and add it to one's endowment). The following papers examine the implications of endowment manipulation: Aumann and Peleg (1974), Hurwicz (1972, 1978), Postlewaite (1979), 
and Thomson (2008)

Now we parametrize strategy-proofness much the same way we parametrized efficiency. Let $\delta \in[0,1]$. Let $(R, \omega) \in \mathcal{E}(\mathcal{R})$ and $i \in N$. Strategy-proofness requires that for each $R_{i}^{\prime} \in \mathcal{R}, \varphi_{i}\left(R_{i}^{\prime}, R_{-i}, \omega\right)$ lie in the lower contour set of $R_{i}$ at $\varphi_{i}(R, \omega)$; i.e., $d\left(\varphi_{i}\left(R_{i}^{\prime}, R_{-i}, \omega\right), L\left(R_{i}, \varphi_{i}(R, \omega) \cap Z_{i}(\omega)\right)=0\right.$. We relax this zero-distance requirement by allowing $\varphi_{i}\left(R_{i}^{\prime}, R_{-i}, \omega\right)$ to lie in a region whose distance from $L\left(R_{i}, \varphi_{i}(R, \omega)\right) \cap Z_{i}(\omega)$, relative to the distance from $L\left(R_{i}, \varphi_{i}(R, \omega)\right) \cap Z_{i}(\omega)$ to $Z_{i}(\omega)$, is $1-\delta$.11

$\boldsymbol{\delta}$-Strategy-proofness: For each $(R, \omega) \in \mathcal{E}(\mathcal{R})$, each $i \in N$, and each $R_{i}^{\prime} \in \mathcal{R}$, $d\left(\varphi_{i}\left(R_{i}^{\prime}, R_{-i}, \omega\right), L\left(R_{i}, \varphi_{i}(R, \omega)\right) \cap Z_{i}(\omega)\right) \leq(1-\delta) d\left(Z_{i}(\omega), L\left(R_{i}, \varphi_{i}(R, \omega)\right) \cap Z_{i}(\omega)\right)$.

As $\delta$ decreases from 1 to $0, \delta$-strategy-proofness expands the set of bundles that an agent can attain with misrepresentation. When $\delta=0$, the latter set is the same as the set of feasible bundles and $\delta$-strategy-proofness has no bite.

Example 2. Illustration of $\delta$-strategy-proofness. Let $M=\{1,2\}$ and $(R, \omega) \in \mathcal{E}(\mathcal{R})$. Let $i \in N$, and suppose that $R_{i}$ and $\varphi_{i}(R, \omega)$ are as specified in Figure 2, Strategyproofness requires that for each $R_{i}^{\prime} \in \mathcal{R}, \varphi_{i}\left(R_{i}^{\prime}, R_{-i}, \omega\right) \in \operatorname{pol}\left[O_{1}, a, b, e\right]$. Now let $\alpha \equiv$ $d\left(Z_{i}(\omega), L\left(R_{i}, \varphi_{i}(R, \omega)\right) \cap Z_{i}(\omega)\right)$. Then 0.7 -strategy-proofness requires that any bundle that agent $i$ obtains by reporting a preference relation lie within $0.3 \alpha$ of $\operatorname{pol}\left[O_{1}, a, b, e\right]$; i.e., for each $R_{i}^{\prime} \in \mathcal{R}, \varphi_{i}\left(R_{i}^{\prime}, R_{-i}, \omega\right) \in \operatorname{pol}\left[O_{1}, a, c, g, f\right]$ (shaded). Similarly, if we impose 0.4-strategy-proofness, then any bundle that agent $i$ obtains by reporting a preference relation should lie within $0.6 \alpha$ of $\operatorname{pol}\left[O_{1}, a, b, e\right]$; i.e., for each $R_{i}^{\prime} \in \mathcal{R}, \varphi_{i}\left(R_{i}^{\prime}, R_{-i}, \omega\right) \in$ $\operatorname{pol}\left[O_{1}, a, d, h, f\right]$. As $\delta \in[0,1]$ decreases, the set of bundles that agent $i$ can achieve expands monotonically, eventually containing all feasible bundles when $\delta=0$.

\section{Impossibility Results}

It is well known that no rule is individually rational, efficient, and strategy-proof. Starting with Hurwicz (1972), a number of works prove this under different restrictions on the preference domain (Dasgupta, Hammond, and Maskin, 1979; Ju, 2003; Schummer,

\footnotetext{
${ }^{11}$ As was the case with $\delta$-efficiency (see Footnote 9), if $\|\omega\|$ is small, even a slight expansion of $L\left(R_{i}, \varphi_{i}(R, \omega)\right) \cap Z_{i}(\omega)$ may contain the set of feasible bundles for agent $i$. Thus, we use $d\left(Z_{i}(\omega), L\left(R_{i}, \varphi_{i}(R, \omega)\right) \cap Z_{i}(\omega)\right)$ as a normalizing factor.
} 
1997; Serizawa and Weymark, 2003; Zhou, 1991). Now we quantify the extent of the impossibility result by replacing efficiency by $\delta$-efficiency and decreasing $\delta$ until the three axioms admit a rule satisfying them. The following theorem says that unless $\delta$-efficiency is vacuous, the three axioms are incompatible. If $\delta=0$, then the no-trade rule, which, for each economy, selects the endowment profile as an allocation, meets the three axioms.

Theorem 1. Let $N=\{1,2\}$. Suppose that $\mathcal{R}_{\text {lin }} \subseteq \mathcal{R}$. For each $\delta \in(0,1]$, no rule defined on $\mathcal{E}(\mathcal{R})$ is individually rational, $\delta$-efficient, and strategy-proof.

Proof. The proof is by means of an example. Let $\delta \in(0,1]$. Suppose, by contradiction, that a rule $\varphi$ defined on $\mathcal{E}(\mathcal{R})$ satisfies the three axioms listed in the theorem. Since there are only two agents, we use the Edgeworth box representation of allocations.

Case 1: There are two commodities; i.e., $M=\{1,2\}$.

(Figure 3) Let $\omega_{1}=(1,0), \omega_{2}=(0,1)$, and $\Omega \equiv \omega_{1}+\omega_{2}$. First, we label some points in the Edgeworth box ${ }^{12}$ Let $\bar{k} \in \mathbb{N}$ be the largest integer such that $2^{\bar{k}-1} \delta \leq 1$ (Figure 3 illustrates the argument for the case $\bar{k}=3)$. Let $K \equiv\{1, \cdots, \bar{k}\}$. For each $R \in \mathcal{R}_{\text {lin }}^{N}$ such that $1<s\left(R_{1}\right)<s\left(R_{2}\right)$, and each $k \in K$, define

$$
\begin{aligned}
a^{k}\left(R_{2}\right) & \equiv\left(1-\frac{2^{k-1} \delta}{s\left(R_{2}\right)}, 2^{k-1} \delta\right) ; \\
b^{k}\left(R_{1}\right) & \equiv\left(1-\frac{2^{k-1} \delta}{s\left(R_{1}\right)}, 2^{k-1} \delta\right) ; \\
c^{k}(R) & \equiv\left(1-\frac{2^{k-1} \delta}{s\left(R_{1}\right)}-\frac{2^{k-1} \delta}{s\left(R_{2}\right)}, 2^{k} \delta\right) ; \\
d^{k}(R) & \equiv\left(1-\frac{1-2^{k-1} \delta}{s\left(R_{1}\right)}-\frac{2^{k-1} \delta}{s\left(R_{2}\right)}, 1\right) ; \text { and } \\
e^{k}(R) & \equiv\left(1-\frac{2^{k-1} \delta}{s\left(R_{1}\right)}-\frac{1-2^{k-1} \delta}{s\left(R_{2}\right)}, 1\right) .
\end{aligned}
$$

Also, define $d^{0}\left(R_{1}\right) \equiv\left(1-\frac{1}{s\left(R_{1}\right)}, 1\right)$ and $e^{0}\left(R_{2}\right) \equiv\left(1-\frac{1}{s\left(R_{2}\right)}, 1\right)$.

In words, first draw (i) the $R_{1^{-}}$and $R_{2}$-indifference curves through $\omega$; and (ii) a square of size $\delta$ whose bottom-right vertex lies at $\omega .{ }^{13}$ The $R_{2}$-indifference curve

\footnotetext{
${ }^{12}$ To lighten notation, we refer to all points using the coordinates with respect to agent 1's origin.

${ }^{13}$ Since we use the Edgeworth box representation, "the $R_{2}$-indifference curve through $\omega$ " is, in fact,
} 


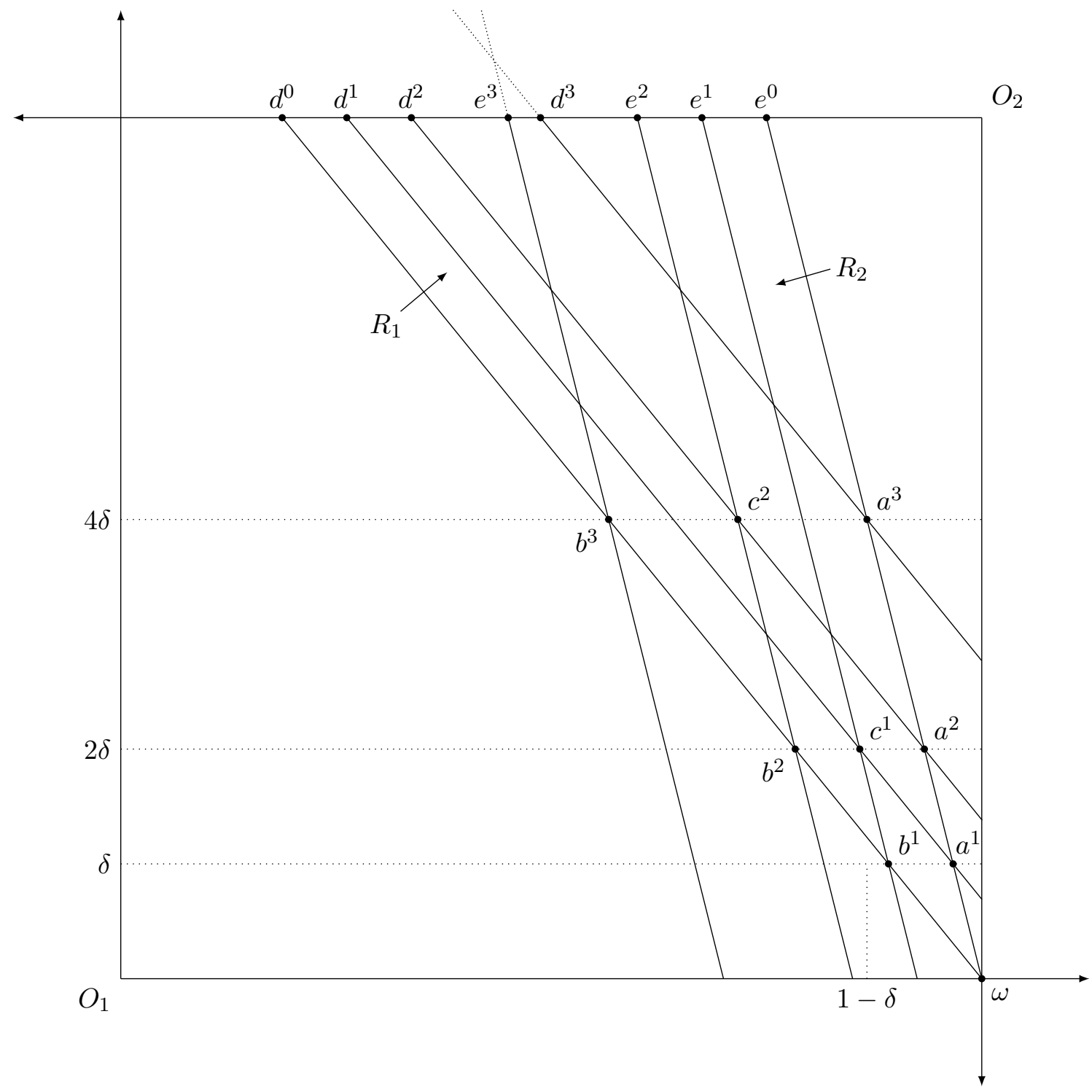

Figure 3: Illustration of Case 1 in the proof of Theorem 1. Let $\delta \in(0,1]$. Let $\omega_{1}=(1,0)$ and $\omega_{2}=(1,0)$. Let $\bar{k} \in \mathbb{N}$ be the largest integer such that $2^{k-1} \delta \leq 1$ (the figure illustrates the argument for the case $\bar{k}=3)$. Let $R \in \mathcal{R}_{\text {lin }}^{N}$ be such that $1<s\left(R_{1}\right)<s\left(R_{2}\right)$. We show by induction that for each $k \in\{1, \cdots, \bar{k}\}, \varphi_{1}(R, \omega) R_{1} a^{k}\left(R_{2}\right)$ and $\varphi_{2}(R, \omega) R_{2}\left(\Omega-b^{k}\left(R_{1}\right)\right.$ ) (in the figure, the arguments of $a^{k}(\cdot), b^{k}(\cdot), c^{k}(\cdot), d^{k}(\cdot)$, and $e^{k}(\cdot)$ are suppressed). In particular, the latter statement is true for $k=\bar{k}$. Because $2^{\bar{k}} \delta>1$, the $R_{1}$-indifference curve through $a^{\bar{k}}\left(R_{2}\right)$ and the $R_{2}$-indifference curve through $b^{\bar{k}}\left(R_{1}\right)$ intersect at a point outside the Edgeworth box. Thus, $\varphi(R, \omega)$ violates feasibility, a contradiction. 
through $\omega$ crosses the top of the square at $a^{1}\left(R_{2}\right)$ and the top of the Edgeworth box at $e^{0}$. Similarly, the $R_{1}$-indifference curve through $\omega$ crosses the top of the square at $b^{1}\left(R_{1}\right)$ and the top of the Edgeworth box at $d^{0}$. Next, the $R_{1}$-indifference curve through $a^{1}\left(R_{2}\right)$ crosses the top of the Edgeworth box at $d^{1}(R)$ and the $R_{2}$-indifference curve through $b^{1}\left(R_{1}\right)$ crosses the top of the Edgeworth box at $e^{1}(R)$. Moreover, the two indifference curves intersect at $c^{1}(R)$. To obtain $a^{2}\left(R_{2}\right)$, move $c^{1}(R)$ horizontally to the right until it lies on the $R_{2}$-indifference curve through $\omega$. Similarly, to obtain $b^{2}\left(R_{1}\right)$, move $c^{1}(R)$ horizontally to the left until it lies on the $R_{1}$-indifference curve through $\omega$. Now the $R_{1}$-indifference curve through $a^{2}\left(R_{2}\right)$ and the $R_{2}$-indifference curve through $b^{2}\left(R_{1}\right)$ intersect at $c^{2}(R)$. Repeating the above argument, we construct the remaining points.

Now we proceed in two steps.

Step 1: For each $R \in \mathcal{R}_{\text {lin }}^{N}$ such that $1<s\left(R_{1}\right)<s\left(R_{2}\right)$, and each $k \in K$, $\varphi_{1}(R, \omega) R_{1} a^{k}\left(R_{2}\right)$ and $\varphi_{2}(R, \omega) R_{2}\left(\Omega-b^{k}\left(R_{1}\right)\right)$.

The proof is by induction. First, let $k=1$. Let $R \in \mathcal{R}_{\text {lin }}^{N}$ be such that $1<s\left(R_{1}\right)<$ $s\left(R_{2}\right)$. Let $x \equiv \varphi(R, \omega)$. Note that $I R(R, \omega) \cap E^{\delta}(R, \omega)=\operatorname{pol}\left[a^{1}\left(R_{2}\right), b^{1}\left(R_{1}\right), d^{0}\left(R_{1}\right), e^{0}\left(R_{2}\right)\right]$. To show that $x_{1} R_{1} a^{1}\left(R_{2}\right)$, suppose, by contradiction, that the $R_{1}$-indifference curve through $x_{1}$ passes through some point $f \equiv\left(f_{1}, f_{2}\right) \in \operatorname{seg}\left(a^{1}\left(R_{2}\right), b^{1}\left(R_{1}\right)\right]$. Let $R_{1}^{\prime} \in \mathcal{R}_{\text {lin }}$ be such that $\frac{\delta}{1-f_{1}}<s\left(R_{1}^{\prime}\right)<s\left(R_{2}\right)$; i.e., typical indifference curves of $R_{1}^{\prime}$ are steeper than the line segment joining $(1,0)$ and $f$, but flatter than those of $R_{2}$. Note that $\varphi_{1}\left(R_{1}^{\prime}, R_{2}, \omega\right) \in I R\left(R_{1}^{\prime}, R_{2}, \omega\right) \cap E^{\delta}\left(R_{1}^{\prime}, R_{2}, \omega\right)=\operatorname{pol}\left[a^{1}\left(R_{2}\right), b^{1}\left(R_{1}^{\prime}\right), d^{0}\left(R_{1}^{\prime}\right), e^{0}\left(R_{2}\right)\right]$. Among the points in the latter set, $b^{1}\left(R_{1}^{\prime}\right)$ uniquely minimizes $R_{1}$, and yet $b^{1}\left(R_{1}^{\prime}\right) P_{1} x_{1}$. Thus, if agent 1 with true preference relation $R_{1}$ faces agent 2 announcing $R_{2}$, he is better off announcing $R_{1}^{\prime}$ than telling the truth, in violation of strategy-proofness. A symmetric argument shows that $x_{2} R_{2}\left(\Omega-b^{1}\left(R_{1}\right)\right)$.

Now suppose that the claim holds for some $k \geq 1$ and that $k+1 \in K$. Let $R \in \mathcal{R}_{\text {lin }}^{N}$ be such that $1<s\left(R_{1}\right)<s\left(R_{2}\right)$. Let $x \equiv \varphi(R, \omega)$. Let $R_{1}^{\prime} \in \mathcal{R}_{\text {lin }}$ be such that $s\left(R_{1}\right)<s\left(R_{1}^{\prime}\right)<s\left(R_{2}\right)$, and we apply the induction hypothesis to $\left(R_{1}^{\prime}, R_{2}, \omega\right)$.

the $R_{2}$-indifference curve through $\omega_{2}$. Throughout the paper, all statements concerning agent 2's indifference curves should be understood this way. Only when comparing bundles according to agent 2's preference relation, we use the coordinate system with respect to agent 2's origin; e.g., we write " $\varphi_{2}(R, \omega) R_{2}\left(\Omega-b^{1}\left(R_{1}\right)\right)$ ", but not " $\varphi_{2}(R, \omega) R_{2} b^{1}\left(R_{1}\right)$ ". 
The $R_{1}^{\prime}$-indifference curve through $a^{k}\left(R_{2}\right)$ crosses the top of the Edgeworth box at $d^{k}\left(R_{1}^{\prime}, R_{2}\right)$ and the $R_{2}$-indifference curve through $b^{k}\left(R_{1}^{\prime}\right)$ crosses the top of the Edgeworth box at $e^{k}\left(R_{1}^{\prime}, R_{2}\right)$. The two indifference curves intersect at $c^{k}\left(R_{1}^{\prime}, R_{2}\right)$. Thus, $\varphi_{1}\left(R_{1}^{\prime}, R_{2}, \omega\right) \in \operatorname{pol}\left[c^{k}\left(R_{1}^{\prime}, R_{2}\right), d^{k}\left(R_{1}^{\prime}, R_{2}\right), e^{k}\left(R_{1}^{\prime}, R_{2}\right)\right]$. Among the points in the latter set, $c^{k}\left(R_{1}^{\prime}, R_{2}\right)$ uniquely minimizes $R_{1}$. If $c^{k}\left(R_{1}^{\prime}, R_{2}\right) P_{1} x_{1}$, then when agent 1 with true preference relation $R_{1}$ faces agent 2 announcing $R_{2}$, he is better off announcing $R_{1}^{\prime}$ than telling the truth, in violation of strategy-proofness. Thus, $x_{1} R_{1} c^{k}\left(R_{1}^{\prime}, R_{2}\right)$. Note that this is true for each $R_{1}^{\prime} \in \mathcal{R}_{\text {lin }}$ such that $s\left(R_{1}\right)<s\left(R_{1}^{\prime}\right)<s\left(R_{2}\right)$. Now construct a sequence of preference relations $\left\{R_{1}^{\nu}\right\}_{\nu \in \mathbb{N}}$ such that (i) for each $\nu \in \mathbb{N}$, $s\left(R_{1}\right)<s\left(R_{1}^{\nu}\right)<s\left(R_{2}\right)$; and (ii) and $\lim _{\nu \rightarrow \infty} s\left(R_{1}^{\nu}\right)=s\left(R_{2}\right)$. Then for each $\nu \in \mathbb{N}$, $x_{1} R_{1} c^{k}\left(R_{1}^{\nu}, R_{2}\right)$, so that $x_{1} R_{1} \lim _{\nu \rightarrow \infty} c^{k}\left(R_{1}^{\nu}, R_{2}\right)=a^{k+1}\left(R_{2}\right)$. A symmetric argument shows that $x_{2} R_{2}\left(\Omega-b^{k+1}\left(R_{1}\right)\right)$, and the claim is established for $k+1$.

\section{Step 2: Concluding.}

Let $R \in \mathcal{R}_{\text {lin }}^{N}$ be such that $1<s\left(R_{1}\right)<s\left(R_{2}\right)$. Let $x \equiv \varphi(R, \omega)$. By Step 1 , for each $k \in K, x_{1} R_{1} a^{k}\left(R_{2}\right)$ and $x_{2} R_{2}\left(\Omega-b^{k}\left(R_{1}\right)\right)$, and in particular, this is true for $k=\bar{k}$. Because $2^{\bar{k}} \delta>1$, the $R_{1}$-indifference curve through $a^{\bar{k}}\left(R_{2}\right)$ and the $R_{2}$-indifference curve through $b^{\bar{k}}\left(R_{1}\right)$ intersect at a point outside the Edgeworth box. Thus, $\varphi(R, \omega)$ violates feasibility, a contradiction.

Case 2: There are more than two commodities; i.e., $M=\{1, \cdots, m\}$, where $m \geq 3$.

(Figure 4 illustrates the argument for the case $m=3$.) Let $\omega_{1}=(1,0, \cdots, 0)$ and $\omega_{2}=(0,1, \cdots, 1)$. It is easy to see that for each $R \in \mathcal{R}_{\text {lin }}^{N}$ such that $r_{1}=\left(r_{11}, 1, \cdots, 1\right)$, $r_{2}=\left(r_{21}, 1, \cdots, 1\right)$, and $1<r_{11}<r_{21}, E(R, \omega)=\left\{x \in Z(\omega)\right.$ : either $x_{11}=0$ or $\left.x_{22}=\cdots=x_{2 m}=0\right\}$ (if $m=3$, then $E(R, \omega)=\operatorname{pol}\left[O_{1}, a, b, c\right] \cup \operatorname{seg}\left[b, O_{2}\right]$ ). Also, $d(Z(\omega), E(R, \omega))=d(\omega, E(R, \omega))$. Thus, we can construct a hypercube of size $\delta$ in the $m$ dimensional Edgeworth box such that (i) one of its vertices lies at $\omega$; and (ii) it does not intersect with the interior of $E^{\delta}(R, \omega)$ (if $m=3$, then $E^{\delta}(R, \omega)$ is the closure of the neighborhood of $E(R, \omega)$ with radius $1-\delta)[14$ Since the counterpart of the latter hypercube in Case 1 is the square of size $\delta$ whose bottom-right vertex lies at $\omega$, we can now proceed as in Case 1.

\footnotetext{
${ }^{14}$ For simplicity, here we identify a feasible allocation with agent 1 's bundle. If we treat the hypercube as an object in $Z(\omega)$, which is a $2 m$ dimensional space, then its size is $\sqrt{2} \delta$.
} 


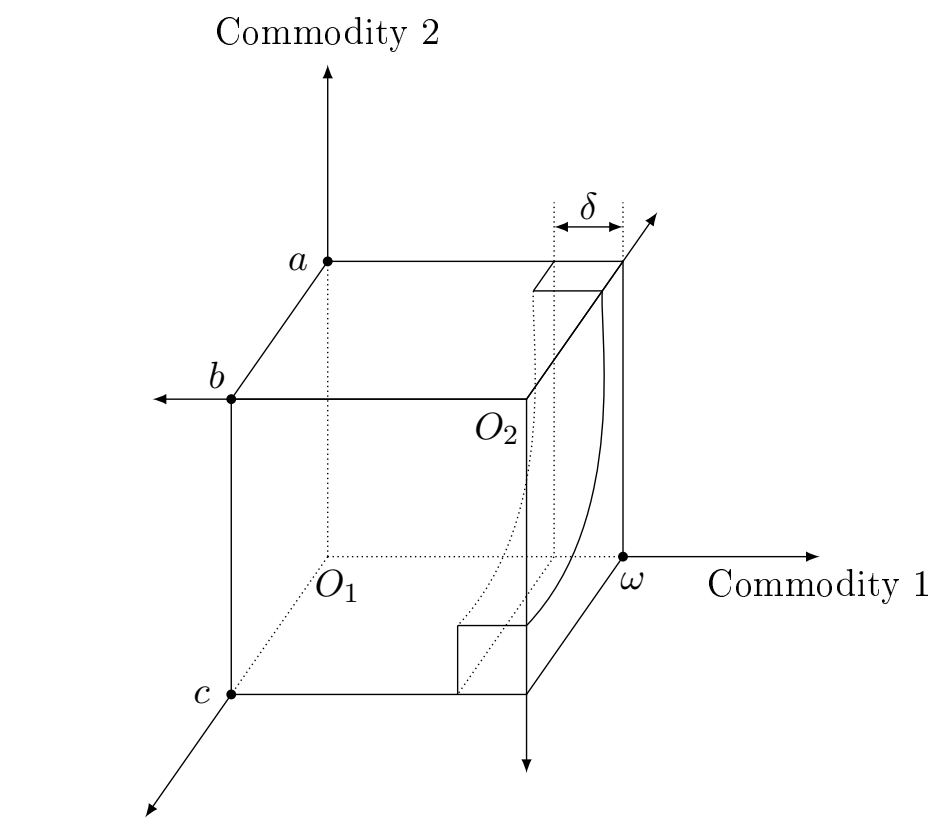

Commodity 3

Figure 4: Illustration of Case 2 in the proof of Theorem $\mathbf{1}$. Assume that $m=3$. Let $\delta \in(0,1]$. Let $\omega_{1}=(1,0,0)$ and $\omega_{2}=(0,1,1)$. For each $R \in \mathcal{R}_{\text {lin }}^{N}$ such that $r_{1}=\left(r_{11}, 1,1\right)$, $r_{2}=\left(r_{21}, 1,1\right)$, and $1<r_{11}<r_{21}$, it follows that $E(R, \omega)=\operatorname{pol}\left[O_{1}, a, b, c\right] \cup \operatorname{seg}\left[b, O_{2}\right]$, and $E^{\delta}(R, \omega)$ is the closure of the neighborhood of $E(R, \omega)$ with radius $1-\delta$. The rest of the argument is similar to that in Case 1.

Next, we replace strategy-proofness by $\delta$-strategy-proofness while retaining individual rationality and efficiency. The next theorem shows that, again, unless $\delta$-strategyproofness is vacuous, the three axioms are incompatible. If $\delta=0$, then we can easily construct an individually rational and efficient rule.

Theorem 2. Let $N=\{1,2\}$. Suppose that $\mathcal{R}_{\text {lin }} \subseteq \mathcal{R}$. For each $\delta \in(0,1]$, no rule defined on $\mathcal{E}(\mathcal{R})$ is individually rational, efficient, and $\delta$-strategy-proof.

Proof. The proof is by means of an example. Let $\delta \in(0,1]$. Suppose, by contradiction, that a rule $\varphi$ defined on $\mathcal{E}(\mathcal{R})$ satisfies the three axioms listed in the theorem. Since there are only two agents, we use the Edgeworth box representation of allocations.

Case 1: There are two commodities; i.e., $M=\{1,2\}$.

(Figure 5) Let $\omega_{1}=(1,0)$ and $\omega_{2}=(0,1)$. Let $\bar{s} \in(0, \delta)$. Let $R \in \mathcal{R}_{\text {lin }}^{N}$ be such 


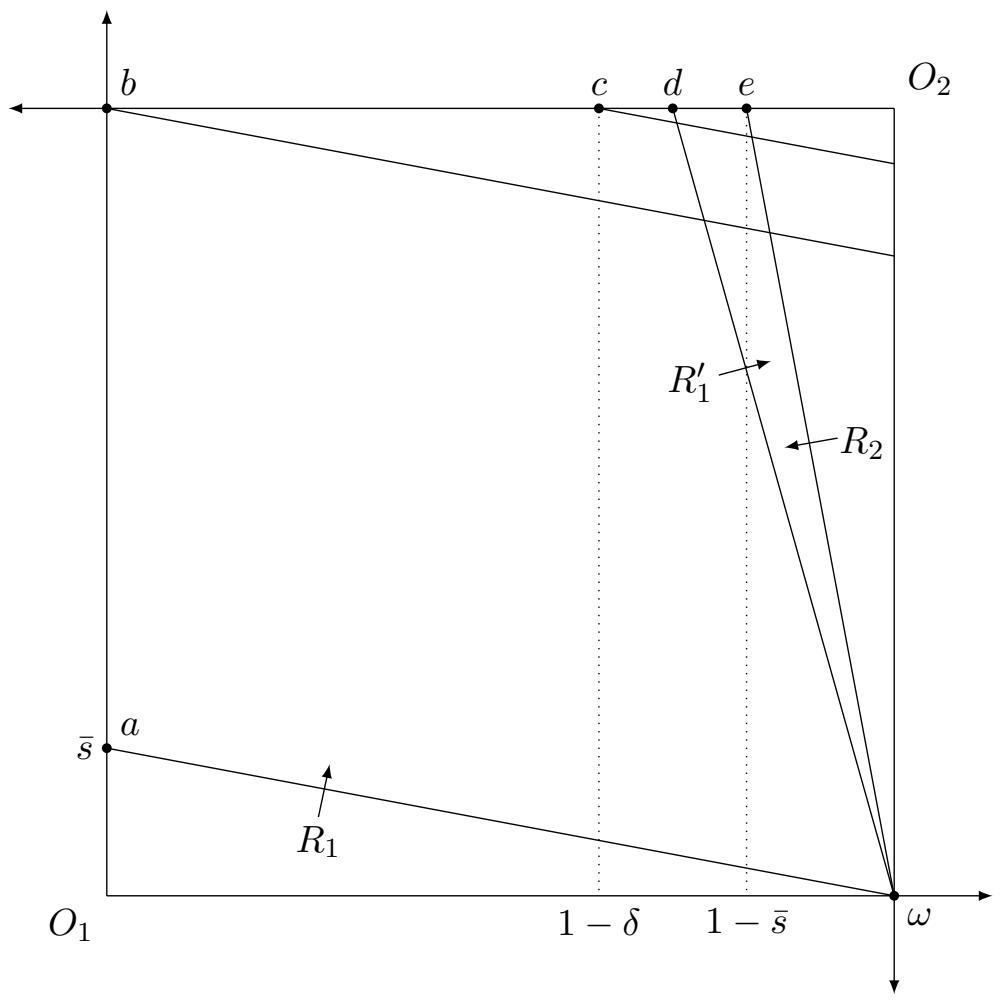

Figure 5: Illustration of Case 1 in the proof of Theorem 2. Let $\delta \in(0,1]$. Let $\omega_{1}=(1,0)$ and $\omega_{2}=(1,0)$. Let $\bar{s} \in(0, \delta)$. Let $R \in \mathcal{R}_{\text {lin }}^{N}$ be such that $s\left(R_{1}\right)=\bar{s}$ and $s\left(R_{2}\right)=\frac{1}{\bar{s}}$. By individual rationality and efficiency, $\varphi(R, \omega) \in \operatorname{seg}[a, b] \cup \operatorname{seg}[b, e]$. Without loss of generality, assume that $\varphi(R, \omega) \in \operatorname{seg}[a, b]$. Then for $R_{1}^{\prime} \in \mathcal{R}_{\text {lin }}$ such that $\frac{1}{\delta}<s\left(R_{1}^{\prime}\right)<\frac{1}{\bar{s}}, \varphi\left(R_{1}^{\prime}, R_{2}, \omega\right) \in \operatorname{seg}[d, e]$, in violation of $\delta$-strategy-proofness.

that $s\left(R_{1}\right)=\bar{s}$ and $s\left(R_{2}\right)=\frac{1}{\bar{s}}$. Let $a \equiv(0, \bar{s}), b \equiv(0,1)$, and $e \equiv(1-\bar{s}, 1)$. Let $x \equiv \varphi(R, \omega)$. By individual rationality and efficiency, $x \in \operatorname{seg}[a, b] \cup \operatorname{seg}[b, e]$. Without loss of generality, assume that $x \in \operatorname{seg}[a, b]$. Let $c \equiv(1-\delta, 1)$. If $x=b$, then by $\delta$-strategy-proofness, for each $R_{1}^{\prime} \in \mathcal{R}, \varphi_{1}\left(R_{1}^{\prime}, R_{2}, \omega\right)$ lies on or below the $R_{1}$-indifference curve through $c$ (note that the ratio of the distance from $O_{2}$ to the $R_{1}$-indifference curve through $c$ to the distance from $O_{2}$ to the $R_{1}$-indifference curve through $b$ is $\delta$ ). Similarly, if $x \in \operatorname{seg}[a, b)$, then for each $R_{1}^{\prime} \in \mathcal{R}, \varphi_{1}\left(R_{1}^{\prime}, R_{2}, \omega\right)$ lies below the $R_{1}$-indifference curve through $c$. Now let $R_{1}^{\prime} \in \mathcal{R}_{\text {lin }}$ be such that $\frac{1}{\delta}<s\left(R_{1}^{\prime}\right)<\frac{1}{\bar{s}}$. Let $d \equiv\left(1-s\left(R_{1}^{\prime}\right), 1\right)$. Then by individual rationality and efficiency, $\varphi_{1}\left(R_{1}^{\prime}, R_{2}, \omega\right) \in \operatorname{seg}[d, e]$, in violation of $\delta$-strategy-proofness. 


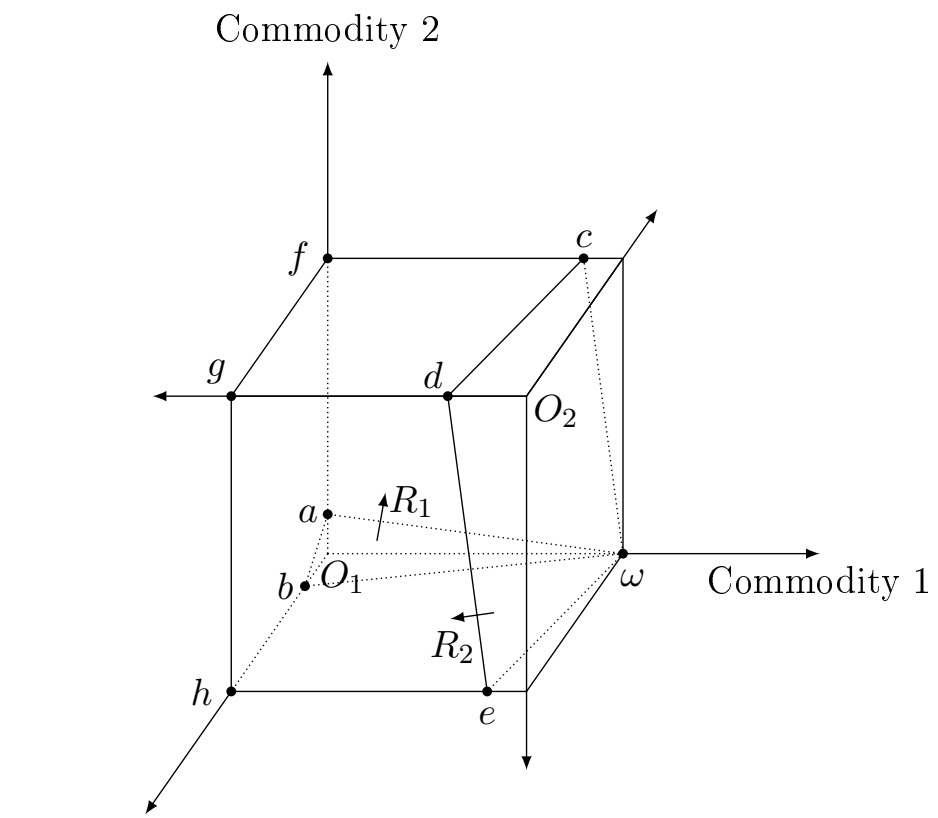

Commodity 3

Figure 6: Illustration of Case 2 in the proof of Theorem 2. Assume that $m=3$. Let $\delta \in(0,1]$. Let $\omega_{1}=(1,0,0)$ and $\omega_{2}=(0,1,1)$. Let $t \in\left(0, \frac{\delta}{2}\right)$. Let $R \in \mathcal{R}_{\text {lin }}^{N}$ be such that $r_{1}=(t, 1, \cdots, 1)$ and $r_{2}=\left(\frac{1}{t}, 1, \cdots, 1\right)$ (the $R_{1}$ - and $R_{2}$-indifference surfaces through $\omega$ are pol $[\omega, a, b]$ and pol $[\omega, c, d, e]$, respectively). By individual rationality and efficiency, $\varphi(R, \omega) \in$ $\operatorname{pol}[a, b, h, g, f] \cup \operatorname{seg}[g, d]$. The rest of the argument is similar to that in Case 1 .

Case 2: There are more than two commodities; i.e., $M=\{1, \cdots, m\}$, where $m \geq 3$.

(Figure 6 illustrates the argument for the case $m=3$.) Let $\omega_{1}=(1,0, \cdots, 0)$ and $\omega_{2}=(0,1, \cdots, 1)$. It is easy to see that for each $R \in \mathcal{R}_{\text {lin }}^{N}$ such that $r_{1}=\left(r_{11}, 1, \cdots, 1\right)$, $r_{2}=\left(r_{21}, 1, \cdots, 1\right)$, and $0<r_{11}<r_{21}, E(R, \omega)=\left\{x \in Z(\omega)\right.$ : either $x_{11}=0$ or $\left.x_{22}=\cdots=x_{2 m}=0\right\}$ (if $m=3$, then $E(R, \omega)=\operatorname{pol}\left[O_{1}, f, g, h\right] \cup \operatorname{seg}\left[g, O_{2}\right]$ ). Let $t \in\left(0, \frac{\delta}{m-1}\right)$. Let $R \in \mathcal{R}_{\text {lin }}^{N}$ be such that $r_{1}=(t, 1, \cdots, 1)$ and $r_{2}=\left(\frac{1}{t}, 1, \cdots, 1\right)$ (if $m=3$, then the $R_{1^{-}}$and $R_{2}$-indifference surfaces through $\omega$ are $\operatorname{pol}[\omega, a, b]$ and $\operatorname{pol}[\omega, c, d, e]$, respectively). Let $x \equiv \varphi(R, \omega)$. Let $B \equiv I R(R, \omega) \cap E(R, \omega)$. Then for each $z \in Z(\omega), z \in B$ if and only if either (i) $z_{11}=0$ and $z_{12}+\cdots+z_{1 m} \geq t$; or (ii) $z_{22}=\cdots=z_{2 m}=0$ and $\left.z_{21} \geq t(m-1)\right\}$ (if $m=3$, then $B=\operatorname{pol}[a, b, h, g, f] \cup \operatorname{seg}[g, d]$ ). By individual rationality and efficiency, $x \in B$. Since the counterpart of $B$ in Case 1 is $\operatorname{seg}[a, b] \cup \operatorname{seg}[b, e]$, we can now proceed as in Case 1. 
Now we connect Theorems 1 and 2 with existing results in the literature. To that end, let us consider an arbitrary parametrization of individual rationality, say $\left.\{\delta \text {-individual rationality }\}_{\delta \in[0,1]}\right]^{15}$ Previous studies show that under some assumptions on the preference domain, if $\varphi$ is efficient and strategy-proof, then for each $\omega \in \mathbb{R}^{M N}$, there is $i \in N$ such that for each $R \in \mathcal{R}^{N}, \varphi_{i}(R, \omega)=\sum_{i \in N} \omega_{i}:{ }^{16}$ see, e.g., Dasgupta, Hammond, and Maskin (1979), Ju (2003), Schummer (1997), and Serizawa (2002). By Schummer (1997), in particular, we obtain the following corollary: for the class of twoagent economies defined on a domain containing linear preference relations, for each $\delta \in(0,1]$, no rule is $\delta$-individually rational, efficient, and strategy-proof.

Thus, we have three impossibility results, each of which involves two axioms in the standard form and one parametrized axiom. Denote by $\delta_{I}, \delta_{E}$, and $\delta_{S}$ the parameters for $\{\delta \text {-individually rationality }\}_{\delta \in[0,1]},\{\delta \text {-efficiency }\}_{\delta \in[0,1]}$, and $\{\delta \text {-strategy-proofness }\}_{\delta \in[0,1]}$, respectively. Consider the $\left(\delta_{I}, \delta_{E}, \delta_{S}\right)$-space in Figure 7 . Since $\left(\delta_{I}, \delta_{E}, \delta_{S}\right) \in[0,1]^{3}$, it can be represented as a point in the three-dimensional unit cube. Hurwicz (1972) shows that (under some assumptions on the preference domain) no rule is individually rational, efficient, and strategy-proof, which corresponds to the non-existence of a rule at point $a \equiv(1,1,1)$.

Most of previous papers study the implications of efficiency and strategy-proofness in terms of fairness. Let us adopt $\delta$-individual rationality as a fairness criterion. Then since $\delta_{E}$ and $\delta_{S}$ are fixed at 1 , their approach can be seen as departing from point $a$ along seg $[a, b]$ in search of an existence result. The corollary above says that at each point in $\operatorname{seg}[a, b)$, we still have an impossibility.

By contrast, our approach is to fix $\delta_{I}$ at 1 and vary $\delta_{E}$ or $\delta_{S}$, one at a time. First, we set $\delta_{I}=\delta_{S}=1$ and decrease $\delta_{E}$, i.e., depart from point $a$ along $\operatorname{seg}[a, c]$, until an existence result emerges. Theorem 1 says that this way of weakening is no more promising: at each point in $\operatorname{seg}[a, c)$, no rule satisfies the three axioms. Next, if we set $\delta_{I}=\delta_{E}=1$ and decrease $\delta_{S}$, i.e., depart from point $a$ along $\operatorname{seg}[a, d]$, then Theorem 2

\footnotetext{
${ }^{15}$ For instance, we may define $\{\delta \text {-individual rationality }\}_{\delta \in[0,1]}$ as follows. First, for each $R_{0} \in \mathcal{R}$ and each $x_{0} \in \mathbb{R}_{+}^{M}$, let $\boldsymbol{U}\left(\boldsymbol{R}_{\mathbf{0}}, \boldsymbol{x}_{\mathbf{0}}\right) \equiv\left\{y_{0} \in \mathbb{R}_{+}^{M}: y_{0} R_{0} x_{0}\right\}$ be the upper contour set of $R_{0}$ at $x_{0}$. Let $\delta \in[0,1]$. For each $(R, \omega) \in \mathcal{E}(\mathcal{R})$ and each $x \in Z(\omega), x$ is $\boldsymbol{\delta}$-individually rational for $(\boldsymbol{R}, \omega)$ if for each $i \in N, d\left(x_{i}, U\left(R_{i}, \omega_{i}\right) \cap Z_{i}(\omega)\right) \leq(1-\delta) d\left(Z_{i}(\omega), U\left(R_{i}, \omega_{i}\right) \cap Z_{i}(\omega)\right)$. A rule is $\delta$-individually rational if for each $(R, \omega) \in \mathcal{E}(\mathcal{R}), \varphi(R, \omega)$ is $\delta$-individually rational for $(R, \omega)$

${ }^{16}$ The identity of agent $i$ may vary depending on the endowment profile chosen. However, once we fix $\omega \in \mathbb{R}_{+}^{M N}$ and treat a preference profile as an economy, the rule $\varphi(\cdot, \omega): \mathcal{R}^{N} \rightarrow \mathbb{R}_{+}^{M N}$ is dictatorial.
} 


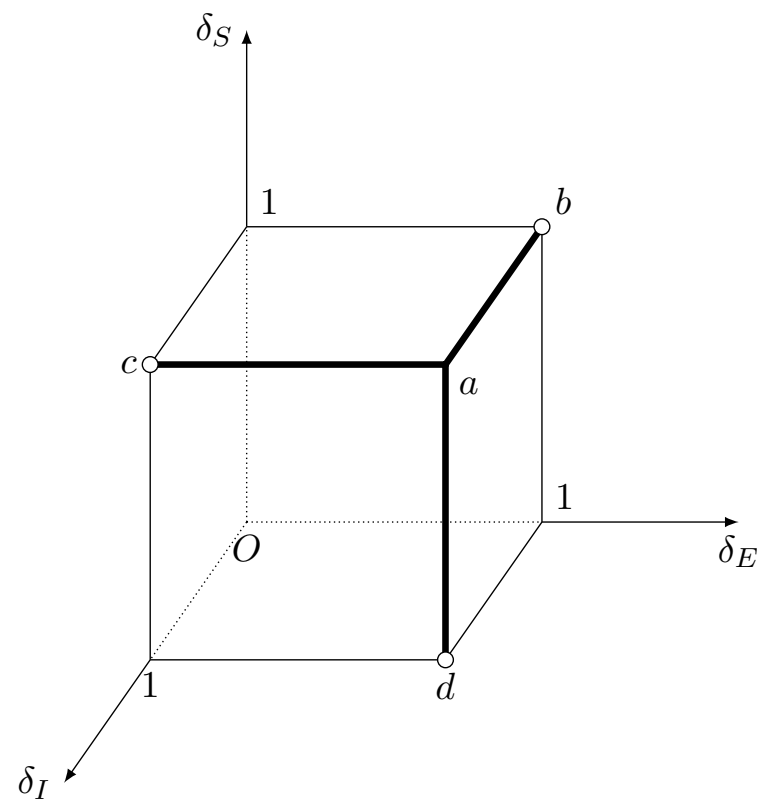

Figure 7: Connection between Theorems 1 and 2 and existing impossibility results. Denote by $\delta_{I}, \delta_{E}$, and $\delta_{S}$ the parameters for $\{\delta \text {-individually rationality }\}_{\delta \in[0,1]},\{\delta \text {-efficiency }\}_{\delta \in[0,1]}$, and $\{\delta \text {-strategy-proofness }\}_{\delta \in[0,1]}$, respectively. Since $\left(\delta_{I}, \delta_{E}, \delta_{S}\right) \in[0,1]^{3}$, it can be represented as a point in the three-dimensional unit cube. Hurwicz (1972) shows that if $\left(\delta_{I}, \delta_{E}, \delta_{S}\right)=(1,1,1)$, i.e., at point $a$ in the figure, no rule is $\delta_{I}$-individually rational, $\delta_{E}$-efficient, and $\delta_{S}$-strategy-proof. Earlier studies show that at each point in $\operatorname{seg}[a, b)$, the three axioms are incompatible. Theorems 1 and 2 show that the same is true on $\operatorname{seg}[a, c)$ and $\operatorname{seg}[a, d)$, respectively.

answers our question: at each point in $\operatorname{seg}[a, d)$, no rule satisfies the three axioms. In sum, the region indicated by thick solid line segments in Figure 7 represents the set of $\left(\delta_{I}, \delta_{E}, \delta_{S}\right)$ at which we have an impossibility.

\section{Robustness of the Results}

Since Theorems 1 and 2 rely on the definition of $\delta$-efficiency and $\delta$-strategy-proofness, one may ask how our results would be affected if the two axioms are defined differently. Now we pursue this question of robustness in two directions.

\section{Using different distance notions}

In defining $\delta$-efficiency and $\delta$-strategy-proofness, we use the standard Euclidean 
distance for convenience, but Theorems 1 and 2 can be proved with different distance notions. We explain how the results can be extended in this regard.

First, concerning Theorem 1, any distance can be used. Let $\hat{d}(\cdot, \cdot)$ be a distance defined on $\mathbb{R}^{M N}$. The distance $\hat{d}(\cdot, \cdot)$ induces the distances between (i) a point and a set in $\mathbb{R}^{M N}$; and (ii) a pair of sets in $\mathbb{R}^{M N}$. Denote those distances by $\hat{d}(\cdot, \cdot)$ as well. Let us now revisit the proof of Theorem 1 . Let $\delta \in(0,1]$. We only consider the two-commodity case for simplicity, but the argument can easily be adapted to the case of three or more commodities. The Euclidean distance allows us to obtain that for each $R \in \mathcal{R}_{\text {lin }}$ such that $1<s\left(R_{1}\right)<s\left(R_{2}\right)$, $\omega$ lies at a positive distance, according to $d(\cdot, \cdot)$, from the set $I R(R, \omega) \cap E^{\delta}(R, \omega)$. For such $R, E(R, \omega)$ is the left and top sides of the Edgeworth box. Therefore, even if $\hat{d}(\cdot, \cdot)$ is used in place of $d(\cdot, \cdot)$ to define $\delta$-efficiency, it still follows that $\hat{d}(Z(\omega), E(R, \omega))=\hat{d}(\omega, E(R, \omega))$, so that $\omega$ lies at a positive distance, according to $\hat{d}(\cdot, \cdot)$, from $I R(R, \omega) \cap E^{\delta}(R, \omega)$. The only difference is that $I R(R, \omega) \cap E^{\delta}(R, \omega)$ may not be a polygon, and the rest of the argument is similar.

Next, concerning Theorem 2 , the following property suffices. Let $\hat{d}(\cdot, \cdot)$ be a distance defined on $\mathbb{R}^{M}$. Again, the distance $\hat{d}(\cdot, \cdot)$ induces the distances between (i) a point and a set in $\mathbb{R}^{M}$; and (ii) a pair of sets in $\mathbb{R}^{M}$. Denote those distances by $\hat{d}(\cdot, \cdot)$. In the proof of Theorem 2, the Euclidean distance is used to deduce an implication of $\delta$-strategyproofness: for each $R_{1}^{\prime} \in \mathcal{R}, \varphi_{1}\left(R_{1}^{\prime}, R_{2}, \omega\right)$ lies on or below the $R_{1}$-indifference surface through $(1-\delta, 1, \cdots, 1)$ (and a symmetric implication for agent 2 ). As long as there is $(\hat{R}, \hat{\omega}) \in \mathcal{E}(\mathcal{R})$ such that $\hat{d}(\cdot, \cdot)$ yields similar implications, the rest of the argument still holds. In particular, this is the case if $\hat{d}(\cdot, \cdot)$ is a "weighted" Euclidean distance; i.e., there is $w \in \mathbb{R}_{++}^{M}$ such that for each pair $a, b \in \mathbb{R}^{M}, \hat{d}(a, b)=\left[\sum_{\ell \in M} w_{\ell}\left(a_{\ell}-b_{\ell}\right)^{2}\right]^{1 / 2}$.

\section{Using different parametrization methods}

As another way of checking the robustness of Theorems 1 and 2, we work with a general parametrization method. We first consider a requirement that a reasonable parametrization should meet, and then show that as long as a parametrization satisfies it, the results similar to Theorems 1 and 2 obtain.

Efficiency and strategy-proofness concern agents' welfare. Therefore, it is best to formulate a parametrization so that a change in welfare is captured precisely. However, the data in our model is not so amenable to such analysis. Each agent only has an 
ordinal preference relation. Unless we take its numerical representation, it is impossible to measure the change in welfare in exact terms. This challenge led us to use the Euclidean distance in our parametrizations. The Euclidean distance is certainly a coarse measure of welfare (the distance between two consumption bundles does not perfectly reflect the difference in welfare that an agent derives from them). Yet, as we argue now, when $\delta$ approaches zero, the imprecision of $\delta$-efficiency and $\delta$-strategy-proofness in capturing welfare changes vanishes.

Recall that Theorem 1 establishes the non-existence of a rule for any $\delta \in(0,1]$. If $\delta$ is sufficiently close to zero, the set of $\delta$-efficient allocations is "almost" the same as the set of feasible allocations. Since preference relations are continuous, this means that we can attain almost all profiles of feasible welfare levels with $\delta$-efficient allocations. In sum, as $\delta$ approaches zero, the idiosyncrasy of the Euclidean distance disappears and $\delta$-efficiency behaves just as well as any appealing parametrization of efficiency does.

To demonstrate this point formally, we first introduce some concepts. Assume, henceforth, that $N=\{1,2\}$ and $\mathcal{R}_{\text {lin }} \subseteq \mathcal{R}$. For each $(R, \omega) \in \mathcal{E}(\mathcal{R})$ and each $x \in$ $Z(\omega), x$ is most inefficient for $(\boldsymbol{R}, \boldsymbol{\omega})$ if (i) $x$ is not efficient; and (ii) there is no $y \in Z(\omega)$ such that $x$ Pareto dominates $y$ for $(R, \omega){ }^{17}$ Let $\hat{\mathcal{E}}(\mathcal{R}) \subseteq \mathcal{E}(\mathcal{R})$ be the class of economies $(R, \omega)$ such that (i) $R \in \mathcal{R}_{\text {lin }}^{N}$; (ii) $r_{1}=\left(r_{11}, 1, \cdots, 1\right), r_{2}=\left(r_{21}, 1, \cdots, 1\right)$, and $1<r_{11}<r_{21}$; and (iii) $\omega_{1}=(1,0, \cdots, 0)$ and $\omega_{2}=(0,1, \cdots, 1)$ (if $m=2$, then $\omega_{1}=(1,0)$ and $\left.\omega_{2}=(0,1)\right)$. The economies in $\hat{\mathcal{E}}(\mathcal{R})$ are the ones used in the proof of Theorem 1 .

Now we define a property that a reasonable parametrization should satisfy. Let $\{\gamma \text {-efficiency }\}_{\gamma \in[0,1]}$ be a parametrization of efficiency. Let $(R, \omega) \in \hat{\mathcal{E}}(\mathcal{R})$. Because $\omega$ is most inefficient for $(R, \omega)$-hence, least desirable in terms of efficiency - we may require that $\omega$ be announced $\gamma$-efficient for $(R, \omega)$ only if $\gamma$-efficiency has no bite. Now fix $\gamma \in(0,1]$. Since $\omega$ is not $\gamma$-efficient for $(R, \omega)$ and since the preference relations are continuous, there is a sufficiently small neighborhood of $\omega$ consisting of allocations that are almost as inefficient as $\omega$. We should also announce these allocations not $\gamma$-efficient. Moreover, since for each economy in $\hat{\mathcal{E}}(\mathcal{R})$, the set of efficient allocations is the same,

\footnotetext{
${ }^{17}$ We embed condition (i) in the definition of most inefficient allocations to exclude efficient allocations that satisfy (ii). An allocation can be efficient and satisfy (ii) because we restrict attention to profiles of bundles whose sums equal the sum of endowments.
} 
the neighborhood of $\omega$ should be independent of the choice of $(R, \omega)$. This argument motivates the following property: for each $\gamma \in(0,1]$ and each $\gamma$-efficient rule $\varphi$, there is $\varepsilon>0$ such that for each $(R, \omega) \in \hat{\mathcal{E}}(\mathcal{R}),\|\varphi(R, \omega)-\omega\| \geq \varepsilon$. We call this property condition $\boldsymbol{C}_{\mathbf{1}}$. The next result shows that condition $C_{1}$ is enough for the impossibility in Theorem 1 .

Theorem 3. Let $N=\{1,2\}$. Suppose that $\mathcal{R}_{\text {lin }} \subseteq \mathcal{R}$. Let $\{\gamma \text {-efficiency }\}_{\gamma \in[0,1]}$ be a parametrization of efficiency satisfying condition $C_{1}$. For each $\gamma \in(0,1]$, no rule defined on $\mathcal{E}(\mathcal{R})$ is individually rational, $\gamma$-efficient, and strategy-proof.

Proof. The proof is by means of an example. Let $\gamma \in(0,1]$. Suppose, by contradiction, that a rule $\varphi$ defined on $\mathcal{E}(\mathcal{R})$ satisfies the three axioms listed in the theorem. Since the parametrization $\{\gamma \text {-efficiency }\}_{\gamma \in[0,1]}$ satisfies condition $C_{1}$, there is $\varepsilon>0$ such that for each $(R, \omega) \in \hat{\mathcal{E}}(\mathcal{R}),\|\varphi(R, \omega)-\omega\| \geq \varepsilon$.

Now let $\omega_{1}=(1,0, \cdots, 0)$ and $\omega_{2}=(0,1, \cdots, 1)$ (if $m=2$, then $\omega_{1}=(1,0)$ and $\left.\omega_{2}=(0,1)\right)$. Let $B \equiv\{x \in Z(\omega):\|x-\omega\| \geq \varepsilon\}$. Then there is $\delta \in(0,1]$ such that for each $R \in \mathcal{R}_{\text {lin }}^{N}$ with $(R, \omega) \in \hat{\mathcal{E}}(\mathcal{R}), B \subseteq E^{\delta}(R, \omega)$. Thus, $\varphi$ is individually rational, $\delta$-efficient, and strategy-proof on $\hat{\mathcal{E}}(\mathcal{R}) 18$ However, the proof of Theorem 1 shows that for each $\delta>0$, no rule is individually rational, $\delta$-efficient, and strategy-proof on $\hat{\mathcal{E}}(\mathcal{R})$, a contradiction.

Next, we turn to the robustness of Theorem 2. The theorem establishes the nonexistence of a rule for any $\delta \in(0,1]$. If $\delta$ is sufficiently close to zero, then $\delta$-strategyproofness allows an agent to attain almost all feasible bundles by manipulating a rule. Since his true preference relation is continuous, this means that he can achieve a welfare level that is arbitrarily close to the maximum welfare level possible for him. Thus, as $\delta$ approaches zero, the role of the Euclidean distance in the construction becomes immaterial and $\{\delta \text {-strategy-proofness }\}_{\delta \in[0,1]}$ behaves in a way that any reasonable parametrization of strategy-proofness should.

\footnotetext{
${ }^{18}$ Let $\mathcal{E}(\mathcal{R})^{*} \subseteq \mathcal{E}(\mathcal{R})$ and let $\varphi$ be a rule defined on $\mathcal{E}(\mathcal{R})$. Let $\delta \in[0,1]$. Then $\varphi$ is individually rational on $\mathcal{E}(\mathcal{R})^{*}$ if for each $(R, \omega) \in \mathcal{E}(\mathcal{R})^{*}, \varphi(R, \omega)$ is individually rational for $(R, \omega)$; $\varphi$ is $\delta$-efficient on $\mathcal{E}(\mathcal{R})^{*}$ if for each $(R, \omega) \in \mathcal{E}(\mathcal{R})^{*}, \varphi(R, \omega)$ is $\delta$-efficient for $(R, \omega)$; and $\varphi$ is strategyproof on $\mathcal{E}(\mathcal{R})^{*}$ if for each $(R, \omega) \in \mathcal{E}(\mathcal{R})^{*}$, each $i \in N$, and each $R_{i}^{\prime} \in \mathcal{R}$ such that $\left(R_{i}^{\prime}, R_{-i}, \omega\right) \in$ $\mathcal{E}(\mathcal{R})^{*}, \varphi_{i}(R, \omega) R_{i} \varphi_{i}\left(R_{i}^{\prime}, R_{-i}, \omega\right)$.
} 
Now we give a formal argument expressing this idea. Assume, henceforth, that $N=$ $\{1,2\}$. Let $\{\gamma \text {-strategy-proofness }\}_{\gamma \in[0,1]}$ be a parametrization of strategy-proofness. Let $\omega_{1}=(1,0), \omega_{2}=(0,1)$, and $\Omega \equiv \omega_{1}+\omega_{2}$. Fix $\gamma \in(0,1]$ and suppose that $\varphi$ is $\gamma$-strategyproof. Consider an arbitrary agent, say $i \in N$. Let $R \in \mathcal{R}_{\text {lin }}^{N}$ be such that $\varphi_{i}(R, \omega) \neq \Omega$. When agent $i$ with true preference relation $R_{i}$ faces agent $j \in N \backslash\{i\}$ announcing $R_{j}$, he does not get all of the endowments, $\Omega$, by telling the truth. Since $\gamma$-strategy-proofness requires $\varphi$ to have at least a minimal form of immunity to manipulation, agent $i$ should not be allowed to attain, by lying, a welfare level that is arbitrarily close to the level that $\Omega$ provides, or in physical terms, a bundle that is arbitrarily close to $\Omega$. This suggests imposing the following property on parametrizations of strategy-proofness: for each $\gamma \in(0,1]$, each $\gamma$-strategy-proof rule $\varphi$, and each $i \in N$, there is $\varepsilon>0$ such that for each $R \in \mathcal{R}_{\text {lin }}^{N}$ with $\varphi_{i}(R, \omega) \neq \Omega$, and each $R_{i}^{\prime} \in \mathcal{R}_{\text {lin }},\left\|\varphi_{i}\left(R_{i}^{\prime}, R_{-i}, \omega\right)-\Omega\right\| \geq$ $\varepsilon\left\|\varphi_{i}(R, \omega)-\Omega\right\|$. We call this property condition $\boldsymbol{C}_{\mathbf{2}}$. The next result shows that condition $C_{2}$ is enough for the impossibility in Theorem 2 .

Theorem 4. Let $N=\{1,2\}$. Suppose that $\mathcal{R}_{\text {lin }} \subseteq \mathcal{R}$. Let $\{\gamma \text {-strategy-proofness }\}_{\gamma \in[0,1]}$ be a parametrization of strategy-proofness satisfying condition $C_{2}$. For each $\gamma \in(0,1]$, no rule defined on $\mathcal{E}(\mathcal{R})$ is individually rational, efficient, and $\gamma$-strategy-proof.

Proof. The proof is by means of an example. Let $\gamma \in(0,1]$. Suppose, by contradiction, that a rule $\varphi$ defined on $\mathcal{E}(\mathcal{R})$ satisfies the three axioms listed in the theorem. Then for each $i \in N$, there is $\varepsilon_{i}>0$ satisfying the requirement of condition $C_{2}$. Let $\varepsilon \equiv$ $\min \left\{\varepsilon_{1}, \varepsilon_{2}\right\}>0$.

Case 1: There are two commodities; i.e., $M=\{1,2\}$.

(Figure 8) Let $\omega_{1}=(1,0), \omega_{2}=(0,1)$, and $\Omega \equiv \omega_{1}+\omega_{2}$. Let $\bar{s} \in(0, \varepsilon)$. Let $R \in \mathcal{R}_{\text {lin }}^{N}$ be such that $s\left(R_{1}\right)=\bar{s}$ and $s\left(R_{2}\right)=\frac{1}{\bar{s}}$. Let $a \equiv(0, \bar{s}), b \equiv(0,1)$, and $e \equiv(1-\bar{s}, 1)$. Let $x \equiv \varphi(R, \omega)$. By individual rationality and efficiency, $x \in \operatorname{seg}[a, b] \cup \operatorname{seg}[b, e]$, so that $x_{1} \neq \Omega$. Without loss of generality, assume that $x \in \operatorname{seg}[a, b]$. Let $c \equiv(1-\varepsilon, 1)$. By $\gamma$-strategy-proofness, for each $R_{1}^{\prime} \in \mathcal{R},\left\|\varphi_{1}\left(R_{1}^{\prime}, R_{2}, \omega\right)-\Omega\right\| \geq \varepsilon\left\|x_{1}-\Omega\right\| \geq \varepsilon$ (if $x=b$, then $\left\|x_{1}-\Omega\right\|=1$; otherwise, $\left.\left\|x_{1}-\Omega\right\|>1\right)$. Now let $R_{1}^{\prime} \in \mathcal{R}_{\text {lin }}$ be such that $\frac{1}{\varepsilon}<s\left(R_{1}^{\prime}\right)<\frac{1}{\bar{s}}$. Let $d \equiv\left(1-s\left(R_{1}^{\prime}\right), 1\right)$. Then by individual rationality and efficiency, $\varphi_{1}\left(R_{1}^{\prime}, R_{2}, \omega\right) \in \operatorname{seg}[d, e]$, a contradiction. 


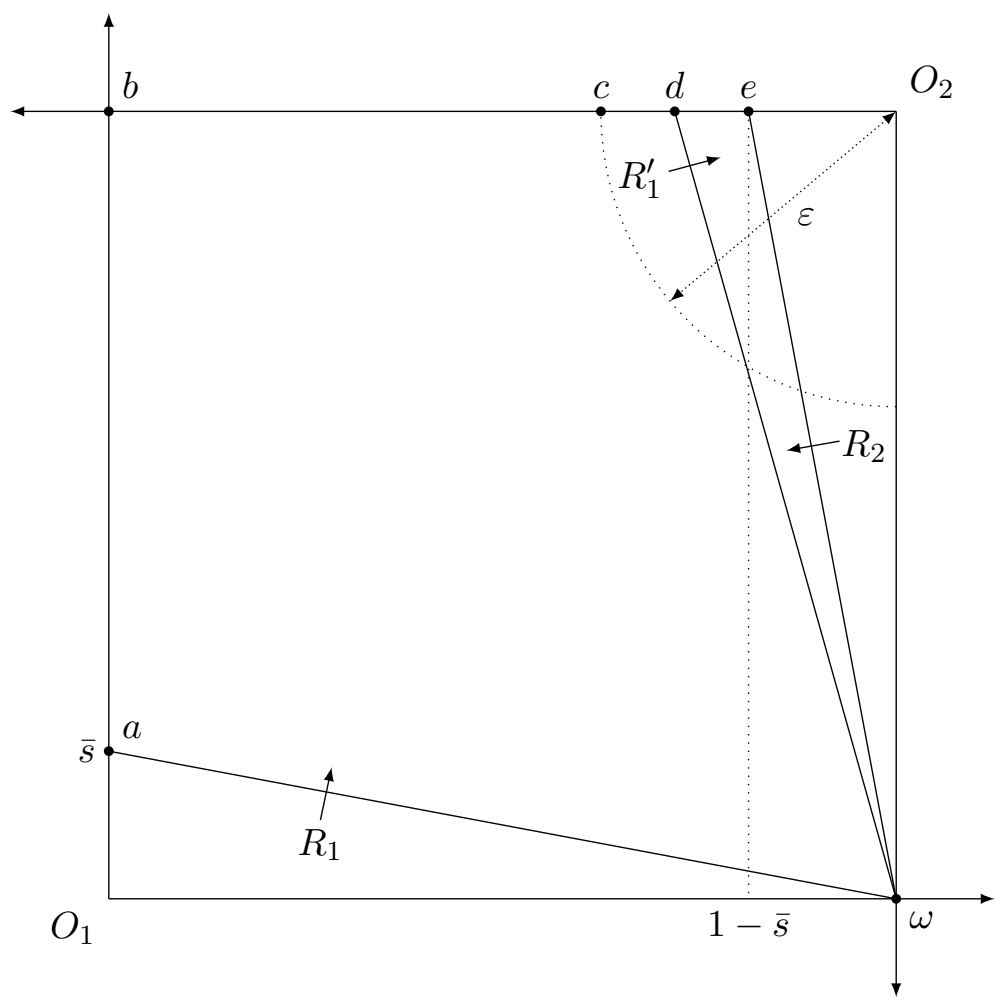

Figure 8: Illustration of Case 1 in the proof of Theorem 4. Let $\gamma \in(0,1]$ and let $\varphi$ be $\gamma$-strategy-proof. Let $\varepsilon>0$ satisfy the requirement of condition $C_{2}$. Let $\omega_{1}=(1,0), \omega_{2}=(0,1)$, and $\Omega \equiv \omega_{1}+\omega_{2}$. Let $R \in \mathcal{R}_{\text {lin }}^{N}$ be as specified in the figure. By individual rationality and efficiency, $\varphi(R, \omega) \in \operatorname{seg}[a, b] \cup \operatorname{seg}[b, e]$. Without loss of generality, assume that $\varphi(R, \omega) \in \operatorname{seg}[a, b]$. By condition $C_{2}$, for each $R_{1}^{\prime} \in \mathcal{R}_{\text {lin }},\left\|\varphi_{1}\left(R_{1}^{\prime}, R_{2}, \omega\right)-\Omega\right\| \geq \varepsilon$. Now let $R_{1}^{\prime}$ be as specified in the figure. By individual rationality and efficiency, $\varphi\left(R_{1}^{\prime}, R_{2}, \omega\right) \in \operatorname{seg}[d, e]$, a contradiction.

Case 2: There are more than two commodities; i.e., $M=\{1, \cdots, m\}$, where $m \geq 3$.

The argument in Case 1 can be adapted to allow for more than two commodities - as in the proof of Theorem 2. We omit the obvious proof.

\section{Concluding Remarks}

We conclude by discussing two issues.

\section{Assumptions on the preference domain.}

Each of our results assumes that the preference domain contains linear preference 
relations. In proofs, we use linear preference relations primarily because they allow us to explicitly calculate the coordinates of the points we are interested in. It is clear that one can derive similar impossibility results, essentially with the same proof, imposing weaker assumptions on the preference domain. For instance, to prove an analog of Theorem 1, it is enough to assume that the preference domain contains the following: (i) a pair of preference relations, say $R_{1}$ and $R_{2}$, such that the $R_{1}$-indifference surface through $\omega$ is everywhere flatter than the $R_{2}$-indifference surface through $\omega$; and (ii) a collection of preference relations, whose indifference surfaces through $\omega$ lie between the $R_{1^{-}}$and $R_{2}$-indifference surfaces through $\omega$ and are arbitrarily close to them.

\section{Identifying the possibility-impossibility boundary in the $\left(\delta_{I}, \delta_{E}, \delta_{S}\right)$-space.}

Figure 7 shows that for each $\left(\delta_{I}, \delta_{E}, \delta_{S}\right) \in \operatorname{seg}[a, b) \cup \operatorname{seg}[a, c) \cup \operatorname{seg}[a, d)$, no rule is $\delta_{I}$-individually rational, $\delta_{E}$-efficient, and $\delta_{S}$-strategy-proof. Yet the region $\operatorname{seg}[a, b) \cup$ $\operatorname{seg}[a, c) \cup \operatorname{seg}[a, d)$ only represents a sufficient condition for the non-existence of a rule. In particular, we do not know whether a rule exists on the faces or in the interior of the unit cube. Thus, one may attempt to identify the possibility-impossibility boundary in the $\left(\delta_{I}, \delta_{E}, \delta_{S}\right)$-space.

While the question is intriguing in itself, it would necessitate a completely new proof technique. Impossibility results are typically proved by a "pathological" counterexample, for which one of the axioms imposed is violated. Therefore, even though no rule exists at a particular point $\left(\delta_{I}, \delta_{E}, \delta_{S}\right)$ in the unit cube, there is no guarantee that the same proof technique would work in a neighborhood of $\left(\delta_{I}, \delta_{E}, \delta_{S}\right)$. Moreover, when it does not work, we cannot be assured that we are near the possibility-impossibility boundary; we still need to check if all examples fail to deliver an impossibility. Since there are a continuum of economies in the domain and a continuum of ways in which agents can misrepresent, this task will prove demanding.

Further, even if a satisfactory answer is available, it would be difficult to extract a meaningful economic content from the answer. The possibility-impossibility boundary characterizes the tradeoff between the three axioms. Since it also serves as a necessary condition for impossibility results, it is very sensitive to the parametrization method chosen. A slight perturbation of the parametrization method may greatly affect the boundary. Therefore, we cannot deduce a robust interpretation from, e.g., the statement 
that a rule exists at $\left(\delta_{I}, \delta_{E}, \delta_{S}\right)$ if and only if $\delta_{I}+\delta_{E}+\delta_{S} \leq 1$.

\section{References}

[1] Aleskerov, F., and E. Kurbanov, 1999, "Degree of manipulability of social choice procedures" in Current Trends in Economics: Theory and Applications (A. Alkan, C. D. Aliprantis, N. C. Yannelis, Eds), Springer, Berlin, Germany.

[2] Aumann, R., and B. Peleg, 1974, "A note on Gale's example", Journal of Mathematical Economics, 1, 209-211.

[3] Dasgupta, P., P. Hammond, and E. Maskin, 1979, "The implementation of social choice rules", Review of Economic Studies, 46, 153-170.

[4] Hurwicz, L., 1972, "On informationally decentralized systems", in Decision and Organization, (C. B. McGuire and R. Radner, Eds), North-Holland, Amsterdam, The Netherlands.

[5] Hurwicz, L., 1978, "On the interaction between information and incentives in organizations", in Communication and Control in Society, (K. Krippendor, Ed), Scientific Publishers, Inc., New York, U.S.A.

[6] Ju, B.-G., 2003, "Strategy-proofness versus efficiency in exchange economies: General domain properties and applications", Social Choice and Welfare, 21, 73-93.

[7] Kelly, J. S., 1993. "Almost all social choice rules are highly manipulable, but a few aren't', Social Choice and Welfare, 10, 161-175.

[8] Maus, S., H. Peters, and T. Storcken, 2007a, "Anonymous voting and minimal manipulability", Journal of Economic Theory, 135, 533-544

[9] Maus, S., H. Peters, and T. Storcken, 2007b, "Minimal manipulability: Anonymity and unanimity", Social Choice and Welfare, 29, 247-269.

[10] Moulin, H., and W. Thomson, 1988, "Can everyone benefit from growth? Two difficulties", Journal of Mathematical Economics, 17, 339-345. 
[11] Postlewaite, A., 1979, "Manipulation via endowments", Review of Economic Studies, 46, 255-262.

[12] Schummer, J., 1997, "Strategy-proofness versus efficiency on restricted domains of exchange economies", Social Choice and Welfare, 14, 47-56.

[13] Serizawa, S., 2002, "Inefficiency of strategy-proof rules for pure exchange economies", Journal of Economic Theory, 106, 219-241.

[14] Serizawa, S., and J. A. Weymark, 2003, "Efficient strategy-proof exchange and minimum consumption guarantees", Journal of Economic Theory, 109, 246-263.

[15] Smith, D., 1999, "Manipulability measures of common social choice functions", Social Choice and Welfare, 16, 639-661.

[16] Thomson, W., 2008, "Borrowing-proofness", mimeo.

[17] Zhou, L., 1991, "Inefficiency of strategy-proof allocation mechanisms in pure exchange economies", Social Choice and Welfare, 8, 247-254. 